\title{
AMPA GluA2 subunit competitive inhibitors for PICK1 PDZ domain: Pharmacophore-based virtual screening, molecular docking, molecular dynamics simulation, and ADME studies
}

\author{
Shravan B. Rathoda,*, Pravin B. Prajapatib,\#,*, Ranjan Palc,\#, Mohmedyasin F. Mansurid \\ aDepartment of Chemistry, Smt. S. M. Panchal Science College, Talod, Gujarat, India \\ ${ }^{b}$ Department of Chemistry, Sheth M. N. Science College, Patan, Gujarat, India \\ 'Department of Medical Genetics, Sanjay Gandhi Post-graduate Institute of Medical Sciences, \\ Lucknow, Uttar Pradesh, India \\ ${ }^{d}$ Department of Microbiology, Smt. S. M. Panchal Science College, Talod, Gujarat, India
}

${ }^{*}$ Corresponding authors:

Shravan B. Rathod- E-mail: shravanathorizon93@gmail.com, Mobile: (+91) 8200040941,

ORCID iD: http://orcid.org/0000-0002-1870-2508

Pravin B. Prajapati- E-mail: pravinprajapati3690@gmail.com, Mobile: (+91) 8000801358,

ORCID iD: https://orcid.org/0000-0003-4752-3821

\#Equal contribution 


\begin{abstract}
PICK1 (Protein interacting with $C$ kinase-1) plays a key role in the regulation of intracellular trafficking of AMPA GluA2 subunit that is linked with synaptic plasticity. PICK1 is a scaffolding protein and binds numerous proteins through its PDZ domain. Research showed that synaptic plasticity is altered upon disrupting the GluA2-PDZ interactions. Inhibiting PDZ and GluA2 binding lead to beneficial effects in the cure of neurological diseases thus, targeting PDZ domain is proposed as a novel therapeutic target in such diseases. For this, various classes of synthetic peptides were tested. Though small organic molecules have been utilized to prevent these interactions, the number of such molecules is inadequate. Hence, in this study, ten molecular libraries containing large number of molecules were screened against the PDZ domain using pharmacophore-based virtual screening to find the best hits for the PDZ domain. Molecular docking and molecular dynamics simulation studies revealed that Hit_ll is a potent inhibitor for the PDZ domain and confirm the allosteric nature of Hit_III. Additionally, ADME analysis suggests the drug-likeness of both Hit_II and Hit_III. This study suggests that tested hits may have potency against the PDZ domain and can be considered effective to treat neurological disorders.
\end{abstract}

\title{
Keywords
}

PICK1 PDZ domain; AMPA GluA2 subunit; Pharmacophore; Virtual screening; Molecular docking; Molecular dynamics simulation 


\section{Introduction}

Protein interacting with $\mathrm{C}$ kinase-1 (PICK1) is a membrane protein and it is present in a wide array of species from $C$. elegans to humans. It is abundantly expressed in brain and testis tissues in humans. In the cellular region, it is found at the perinuclear site and the neural presynaptic and postsynaptic sections of the Central nervous system (CNS) (Xu \& Xia, 2007). PICK1 contains two vital domains, membrane binding N-BAR (Bin/amphiphysin/rvs) and PDZ (PSD-95/Dlg/ZO1) which binds to the PDZ motifs of other proteins (Herlo et al., 2018; Li et al., 2016). The PDZ domain of PICK1 interacts with various transport proteins, neurotransmitter receptors, and other enzymes (Erlendsson et al., 2014; Xu \& Xia, 2007). Additionally, PDZ is not only a promising drug target for the neurological disorders but also for the viral infections and various kinds of cancers. It is involved in numerous other processes such as DNA replication, extracellular matrix organisation, gene expression, hemostasis, immune responses, and metabolism (Christensen et al., 2019). Since PICK1 is involved in the regulation of proteins that are linked with neuropsychiatric and neurological conditions, it can be considered a potential target for novel therapeutics (Thorsen et al., 2010).

The disruption of protein-protein interactions implicated in cell-cell adhesion, cell death, signal transduction, and other biomolecular processes by small organic molecules can solve many biological challenges for numerous diseases (Arkin \& Wells, 2004; Berg, 2008; Wells \& McClendon, 2007). PDZ domain is involved in therapeutically targeted protein-protein interactions (Blazer \& Neubig, 2009; Dev, 2004; Houslay, 2009) and it helps cellular trafficking and providing scaffolding sites for large assembly forming proteins inside the cell. This domain identifies the C-terminal region of interacting proteins to facilitate further functions and it is highly expressed in eukaryotes (Kornau et al., 1993; Kim et al., 1995; Songyang et al., 1997). Research shows that PICK1 plays a vital role in different forms of synaptic plasticity (Daw et al., 2000; Gardner et al., 2005; Terashima et al., 2008; Xia et al., 2000) involving LTD (Long- term depression) and LTP (Long-term potentiation) through its binding with a-Amino-3-hydroxy5-methyl-isoxazole-4-propionic acid (AMPA) receptors and, it has also been reported as therapeutic target for pain alleviation (Garry et al., 2003), brain ischemia (Bell et al., 2009; Dixon et al., 2009), and cocaine addiction (Bellone \& Lüscher, 2006). PDZ domain has multiple protein partners in the nervous system thus, disruption of these protein-protein interactions leads to the beneficial impacts on the treatment of neural disorders (Khan \& Lafon, 2014). Glutamate receptor (GluA2) subunit of AMPA interacts with the PDZ domain of PICK1 through its C-terminal which is necessary for the AMPA internalization in the cell (Hanley \& Henley, 2005). This enhanced internalization of AMPA through GluA2 mediated mechanism leads to further synaptic depression by Amyloid-beta (A $\beta$ ) which results in a reduction of dendritic spine density (Hsieh et al., 2006). Study showed that small molecule inhibitors mitigate the effects of $A \beta$ on synapse through disrupting PDZ-GluA2 interactions which indicates the role of these interactions as vital to $A \beta$ effects on synaptic functions (Alfonso et al., 2014). Hence, preventing the interactions between AMPA and PICK1 by small molecules can be considered a significant approach to treat diseases and disorders. 
To target the PDZ domain, researchers have designed and investigated short peptides, modified peptides, cyclic peptides, and peptidomimetics but due to their restricted cell permeability, results were not quite promising (Ducki \& Bennett, 2009). Moreover, Bach and his team (Bach et al., 2012) synthesized dimeric peptide that showed high binding affinity $(K i=4.6 \mathrm{nM})$ with the PDZ domain of PICK1. Sharma et al. also screened the peptide libraries against PDZ-3 domain computationally and identified the promising peptides for the PDZ domain (Sharma \& Siddiqi, 2019). But, these inhibitors have poor potency, less selectivity, and distribution problems (Lin et al., 2018). To overcome these challenges, researchers have started to synthesize non-peptide small organic molecules against the PDZ domains (Bach et al., 2010; Marcotte et al., 2018; Thorsen et al., 2010; Wang et al., 2021). Thorsen et al. used Fluorescence polarization (FP) based screening of 43,380 small organic molecules and got promising results targeting this domain (Thorsen et al., 2011).
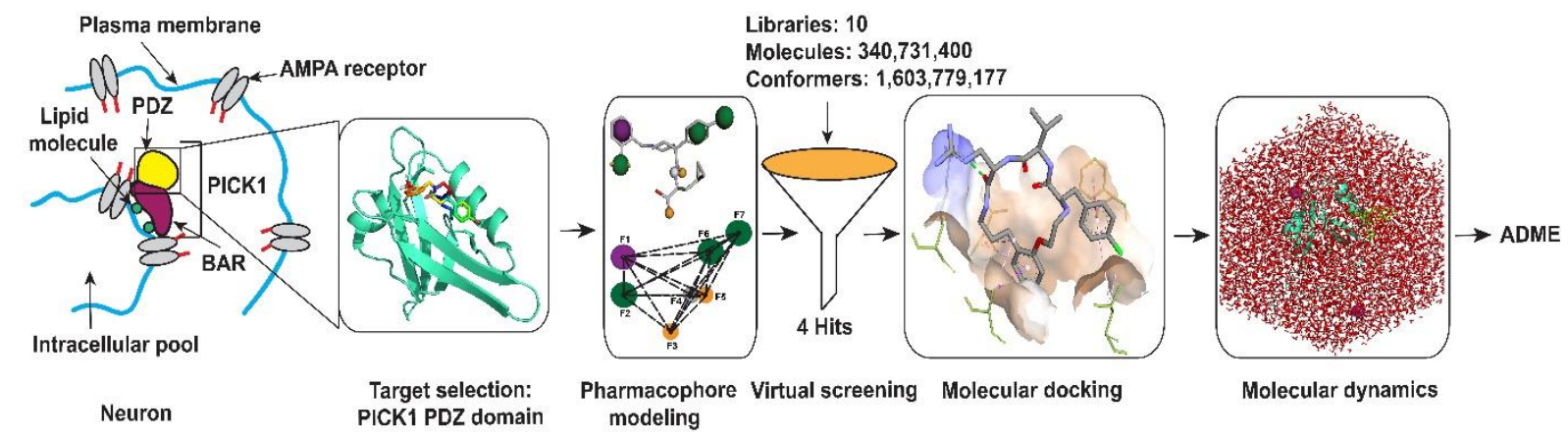

Molecular dynamics

Figure 1. Methodology schema used in this study to identify the best hits against the PICK1 PDZ domain. PICK1 PDZ domain with its inhibitor BQA was retrieved from the PDB database. Then, PDZ domain and BQA were uploaded to the Pharmit, a pharmacophore-based virtual screening web server. Pharmacophore was constructed using seven features of the BQA inhibitor. Then, ten molecular libraries were screened against the PDZ domain. Obtained four best hits were further analyzed for the binding affinity towards the PDZ domain using DockThor molecular docking web server. Finally, all-atom 50 ns molecular dynamics simulation was performed using the GROMACS 2020.1 tool to investigate the dynamic behavior of protein-ligand complexes along with control (BQA) complex. Finally, four hits and control (BQA) were analyzed for drug-likeness using Swiss ADME web server.

We still have a very few candidates that inhibit the PDZ domain compared to the availability of small molecule databases. Thus, in this present study, we utilized Computer-aided drug design (CADD) tools to screen a vast number of small organic molecules and their conformers to identify potential hits for the PDZ domain. Computational analysis spotted four best hits that shows efficient binding towards PDZ. MD analysis showed that Hit_II is a potent inhibitor against PDZ domain and Hit_III, allosterically inhibits the AMPA GluA2 peptide binding at the PDZ binding pocket. Additionally, through ADME analysis, it was confirmed that Hit_II and Hit_III bith show druglikeness. Figure 1 represents the workflow of present study.

\section{Materials and methods}

\section{Protein preparation}

The crystal structure of PICK1 PDZ domain (PDB ID: 6AR4) (Lin et al., 2018) with its inhibitor (BQA: N[4-(4-Bromophenyl)-1-\{[2-(trifluoromethyl)phenyl]methyl\}piperidine-4-carbonyl]-3-cyclopropyl-Lalanine) was retrieved from the Protein data bank (PDB) (https://www.rcsb.org/). It is in a dimeric form 
so, one monomer with its ligand and solvent molecules were deleted from the structure during protein preparation using PyMOL v2.4.1 (Schrödinger LLC., 2010). The missing residues (a.a.: 1-18 \& 105109) were added using MODELLER AutoModel class (Webb \& Sali, 2016) and implicit hydrogens were added in PyMOL. To determine the protein surface property, Electrostatic potential (ESP) was computed using eF-surf-PDBj (https://pdbj.org/eF-surf/top.do) web server. To probe the allosteric nature of Hit_III, $7 \mathrm{~ns}$ frame from the PDZ-Hit_III complex simulation was obtained and further subjected to docking with AMPA GluA2 peptide.

\section{Structure-based pharmacophore modeling and virtual screening}

Pharmacophore-based small molecule virtual screening was carried out using Pharmit web server (Sunseri \& Koes, 2016) available at http://pharmit.csb.pitt.edu/. Literature review suggests that a pharmacophore can be constructed from single inhibitor bound to the protein by considering its interacting features for the virtual screening (Pant et al., 2020; Singh et al., 2020). Hence, For the construction of the pharmacophore model, a prepared protein with its BQA ligand was uploaded to the server. Out of nine features, one feature (hydrogen acceptor) at the carboxylic acid group of BQA was excluded, and remaining parameters were kept unchanged for the pharmacophore modeling. This pharmacophore was further utilized for virtual screening of ten chemical libraries with having $340,731,400$ molecules and 1,603,779,177 conformers (Table 1). After the virtual screening, four hits (RMSD $\leq 4.5 \AA$ ) were identified and their structures (SDF format) were saved for further analysis.

\section{Ligand preparation}

Identified four hits in the virtual screening were opened in Avogadro (Hanwell, et al., 2012) and, implicit hydrogens were added to the structures. Further, energy minimization (MMFF94s force field (Halgren, 1996) \& steepest descent algorithm (Chen et al., 2013)) was performed using Auto Optimization Tool in Avogadro. Optimized structures were saved as Sybyl Mol2 format for molecular docking and dynamics analyses. Additionally, AMPA GluA2 peptide sequence was uploaded during the job submission at HPEPDOCK (Zhou et al., 2018) web server for the peptide docking with the $7 \mathrm{~ns}$ frame of PDZ domain from the previous simulation.

Table 1. Molecular libraries screened against the PDZ domain at Pharmit web server.

\begin{tabular}{lcc}
\hline \multicolumn{1}{c}{ Library } & Molecules & Conformers \\
\hline MCULE-ULTIMATE & $126,471,502$ & $378,880,344$ \\
PubChem & $93,067,404$ & $450,708,705$ \\
ChemSpace & $50,181,678$ & $250,205,463$ \\
MCULE & $45,045,153$ & $222,427,706$ \\
ZINC & $13,190,317$ & $123,399,574$ \\
MolPort & $7,719,859$ & $110,832,826$ \\
LabNetwork & $1,794,286$ & $22,051,020$ \\
CHEMBL25 & $1,752,844$ & $23,136,925$ \\
ChemDiv & $1,456,120$ & $21,562,497$ \\
NCI Open Chemical Repository & 52,237 & 574,117 \\
\hline
\end{tabular}




\section{Molecular docking}

To investigate the binding affinity of previously obtained four hits (Hit_I, Hit_II, Hit_III, and Hit_IV) along with control (BQA), molecular docking of PICK1 PDZ domain with these small organic molecules was performed at a widely used flexible docking web server, DockThor (Santos et al., 2020) available at (https://dockthor.Incc.br/v2/). To run molecular docking, prepared protein (PDB ID: 6AR4) and hits were uploaded to the server. A predefined binding pocket center $(x, y, z)=-15.3 \AA, 6.4 \AA, 5.0 \AA$ with size ( $x$, $y, z)=20 \AA, 20 \AA, 20 \AA$ was set and default options (a. 12 docking runs, b. 500,000 evaluations per docking run, c. population of 750 individuals, and d. maximum of 20 cluster leaders on each docking run) were kept unchanged. For each run, DockThor gives the best pose based on the binding affinity and clustering probability of ligand. Results were downloaded for further analysis. Finally, 2D and 3D interactions were obtained using Discovery Studio v.20.1 (BIOVIA, Dassault Systèmes, Discovery Studio Visualizer, 20.1.0.19295, San Diego: Dassault Systèmes, 2020). Further, to investigate the allosteric behavior of Hit_III, 7 ns frame of PDZ-Hit_III complex was extracted from the trajectory and AMPA GluA2 peptide was docked with the complex using HPEPDOCK web server (Zhou et al., 2018) available at http://huanglab.phys.hust.edu.cn/hpepdock/. During the job submission, template-based docking option was applied. To validate the docking tool, DockThor, redocking was performed and RMSDs were calculated using LigRMSD tool (Velázquez-Libera et al., 2020) available at https://ligrmsd.appsbio.utalca.cl/. The overall RMSD was observed below $0.3 \AA$ (BQA: $0.02 \AA$, Hit_l: $0.27 \AA$, Hit_II: $0.08 \AA$, Hit_III: $0.00 \AA$, and Hit_IV: $0.00 \AA ̊$ ) which indicates the reproducibility of docking results.

\section{Molecular dynamics (MD) simulation}

To probe the effects of ligand on protein dynamics, molecular dynamics simulation was performed using GROMACS 2020.1 version (Abraham et al., 2015) on Linux (Ubuntu 2020.11) system. MD simulations were performed for apo form (PDZ), control (PDZ-BQA), and four hits (PDZ-Hit_I to Hit_IV). MD simulation was carried out using CHARMM36 force field (Best et al., 2012; MacKerell et al., 1998) updated version (charmm36-feb2021) and TIP3P water model (Jorgensen et al., 1983). CHARMM general force field (CGenFF) (Allouche, 2012; Yu et al., 2012) (https://cgenff.umaryland.edu/) was employed to build ligand topologies. Protein (PDZ) and its complexes (PDZ-Hit_I, PDZ-Hit_II, PDZ-Hit_III, PDZ-Hit_IV, and PDZ-BQA) were placed in the center of the dodecahedron box with a $10 \AA$ from the box edges. Additionally, to probe the allosteric nature of Hit_III, two other simulations for PDZ-GluA2 and PDZ-GluA2-Hit_III complexes were also carried out. Positive $\left(\mathrm{Na}^{+}\right)$and negative $\left(\mathrm{Cl}^{-}\right)$ions were added by substituting solvent molecules to the system wherever they were needed to neutralize the whole system. To reduce steric clashes into the system, energy minimization was performed using the steepest descent algorithm (Chen et al., 2013) with Verlet cut-off scheme (Verlet, 1967) and Particle mesh Ewald (PME) (Darden et al., 1993) long-range electrostatic. During energy minimization, a maximum number of steps (nsteps) were 50,000, and system approaching minimum energy was $10 \mathrm{~kJ} / \mathrm{mol}$. Further, NVT and NPT based equilibrations were carried out at $300 \mathrm{~K}$ temperature and for $100 \mathrm{ps}(50,000$ steps $)$ with a 2 fs time step gap. Finally, MD 
simulation was performed for 50 ns using leap-frog integrator (Lippert et al., 2007), Verlet cutoff scheme, and PME at $300 \mathrm{~K}$ (modified Berendsen thermostat (Berendsen et al., 1984)) and 1 bar pressure (Parrinello-Rahman method (Parrinello \& Rahman, 1981)). All the bonds were constrained with LINCS (Linear constraint solver) algorithm (Hess et al., 1997). Required Python script and CHARMM36 force field file were downloaded from the MacKerell lab website (http://mackerell.umaryland.edu/charmm ff.shtml\#gromacs). MD trajectories were analyzed for various parameters such as Root mean square deviation (RMSD), Root mean square fluctuation (RMSF), Solvent accessible surface area (SASA), Radius of gyration (Rg), and intramolecular hydrogen bonds in PDZ and intermolecular hydrogen bonds in the complexes. Furthermore, to superimpose the BQA and hits in the binding pocket, frames from every $10 \mathrm{~ns}$ were extracted from the trajectory and aligned in PyMOL. PDZ domain was deleted from each complex and $B Q A$ and hits were saved in MOL format. LigRMSD web tool was used to superimpose the BQA and hits. The average RMSD was calculated with a reference to $1 \mathrm{~ns}$ frame for all the ligands except Hit_III. The average RMSD of Hit_III was calculated in the allosteric pocket with reference to the 10 ns frame.

\section{ADME analysis}

The SwissADME web server (Daina et al., 2017) available at http://www.swissadme.ch/ was used to evaluate the ADME properties of studied four hits along with control (BQA). It is widely used to assess the ADME properties like physiochemical, lipophilicity, water solubility, pharmacokinetics, and druglikeness of potential drug candidates. Additionally, we also ran BOILED-Egg model (Daina \& Zoete, 2016) to probe the passive Human gastrointestinal absorption (HIA), Blood-brain barrier (BBB), and the Permeability-glycoprotein (P-gp) substrate-non-substrate identification. This model is an extended feature of SwissADME. Furthermore, we have included bioavailability radar which explains six parameters such as lipophilicity, size, polarity, solubility, flexibility, and saturation to detect the druglikeness of compounds.

\section{Results and discussion \\ Catalytic site of PICK1 PDZ domain}

The interactions of the PDZ domain with the C-termini of NMDA (N-methyl-D-aspartate) and with the AMPA GluA2 subunit are thought to play central role in synaptic plasticity (Kim et al., 2001). Lin et al. (Lin et al., 2018) have solved the crystal structure of the PICK1 PDZ domain with its inhibitor (BQA) and they observed that inhibitor binds (IC50: $0.573 \mu \mathrm{M}$ ) to the AMPA GluA2 subunit binding pocket of the PDZ domain. Figure 2A-C illustrates the $\mathrm{H}$-bond and hydrophobic surface representations and 2D interactions of $B Q A$ with PDZ domain interacting residues. The protein surface analysis in the form of Electrostatic potential (ESP) showed that the binding pocket $(x, y, z)=(-15.3 \AA, 6.4 \AA, 5.0 \AA)$ is hydrophobic (Figure 2D-E) in nature where BQA and AMPA GluA2 peptide bind. BQA has three main hydrophobic regions (Figure $3 \mathrm{~B}$ ), which are designated by R1, R2, and R3. 


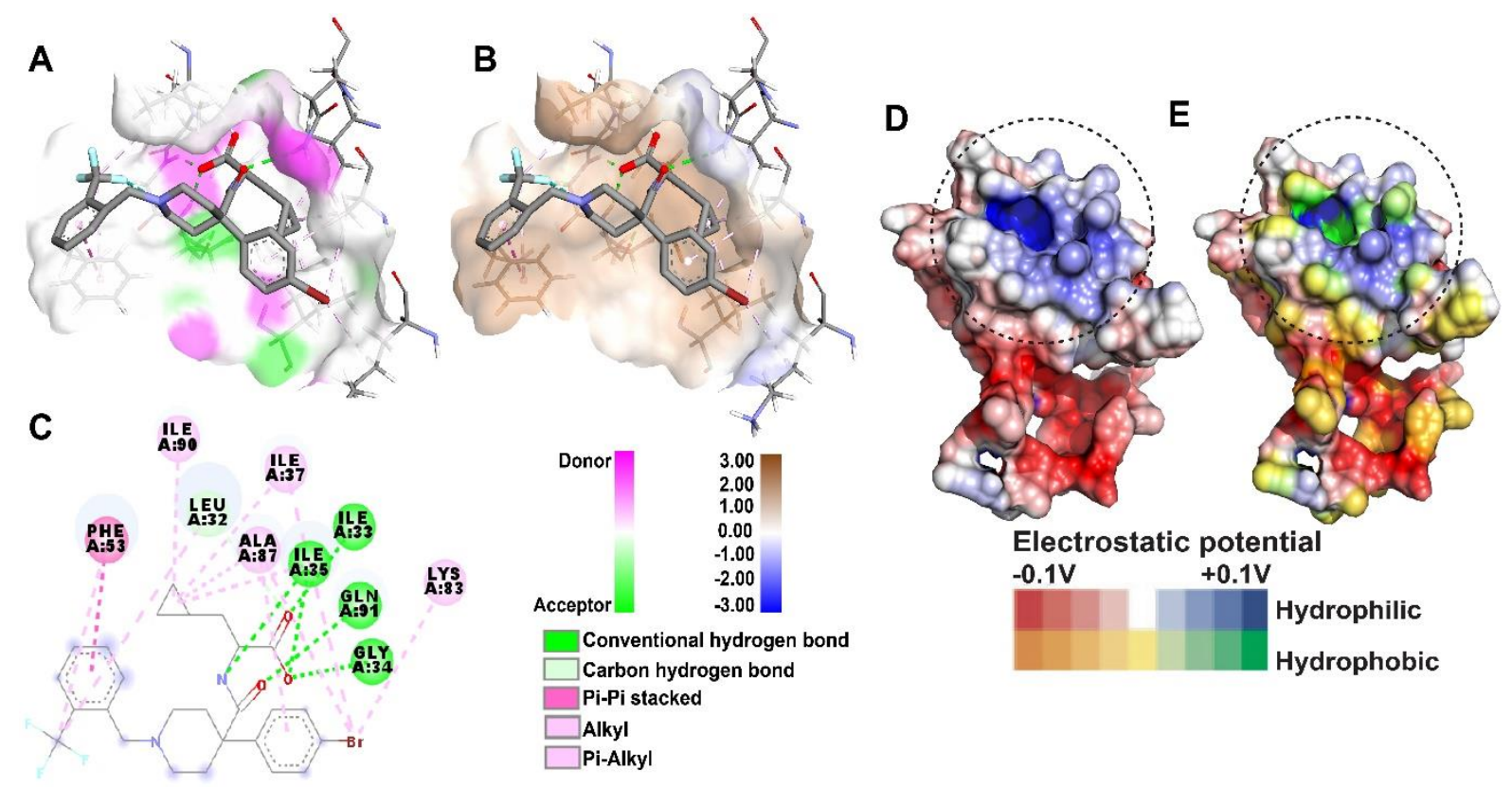

Figure 2. BQA binding pose inside the PDZ domain and 2D interactions. (A) Hydrogen bond surface representation (B) Hydrophobic surface representation, and (C) 2D interactions (D) Electrostatic potential (ESP) surface representations of the PDZ domain. Black dashed circles indicate the ligand-binding pocket on the PDZ domain which is hydrophobic in nature.

These three sites show dominant interactions with the different amino acid residues of the PDZ domain. BQA interacts with the PDZ domain through multiple hydrogen bonds and hydrophobic contacts. Hydrogen bonds are formed by the carboxylic acid and amide groups whereas hydrophobic interactions are formed by the remaining sites such as cyclopropyl ring, trifluoromethyl group, a bromine atom, and aromatic rings present in the BQA. The binding pocket has Asp28, Leu32, Ile33, Gly34, Ile35, Phe53, Thr56, Lys83, and Ala87 residues. R1 (-CF3 group) of BQA is surrounded by Asp28, Leu32, and Thr56, $\mathrm{R} 2\left(-\mathrm{C}_{6} \mathrm{H}_{4} \mathrm{Br}\right.$ group) by Lys83 and Ala87, and $\mathrm{R} 3$ (cyclopropane ring, $\left.\mathrm{R}-\mathrm{CH}-\left(\mathrm{CH}_{2}\right)_{2}\right)$ by lle33, Gly34, and Ile35 neighboring residues. During pharmacophore construction, the R3 hydrophobic pocket was ignored to screen the libraries.

\section{Structure-based pharmacophore modeling and virtual screening}

To screen the large number of compounds against the PICK1 PDZ domain, pharmacophore was constructed by selecting specific ligand features that interact with the specific residues of the PDZ domain. The aim was to find the best-fit hits for the PDZ domain and consequently, a maximum number of features (seven) were kept activated during the virtual screening. 


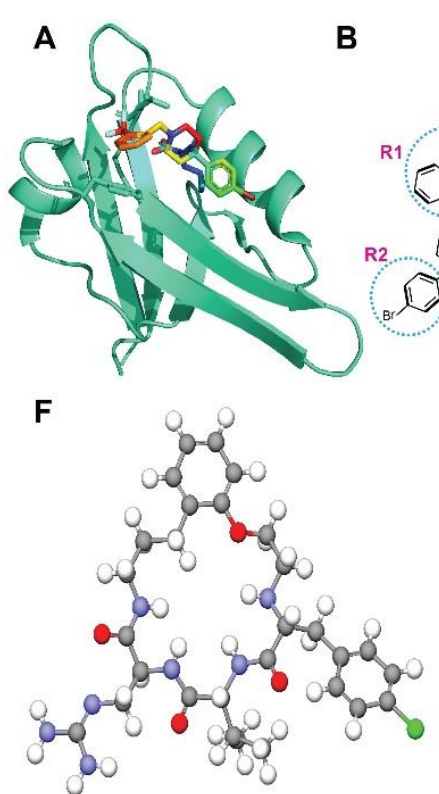

Hit_I

J

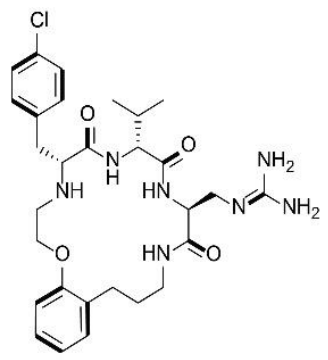

Hit_I

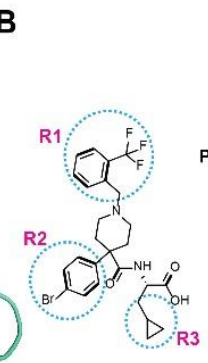

G

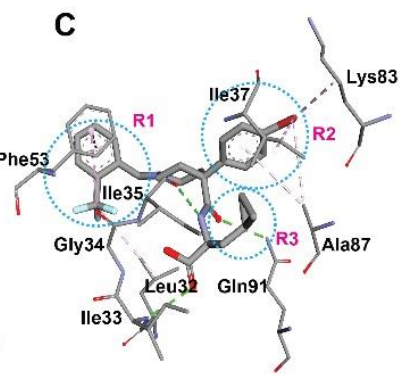

H

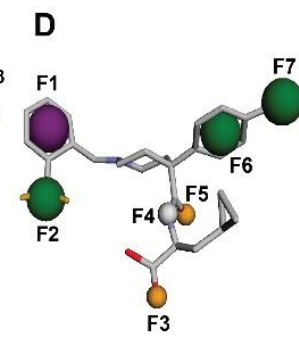

I

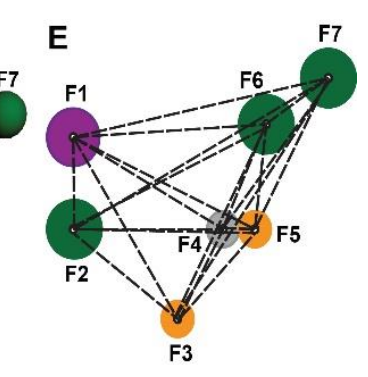

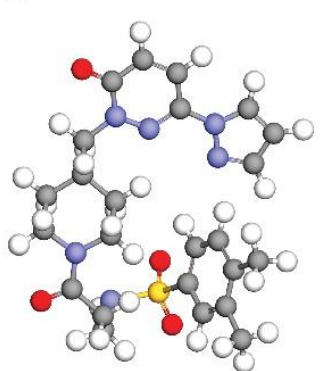

Hit_II

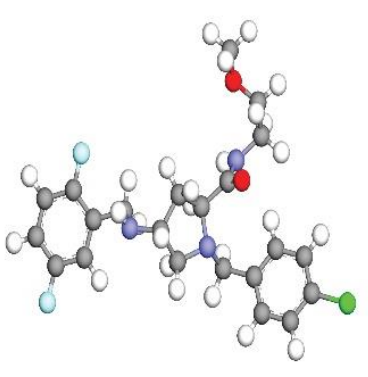

Hit_III

K

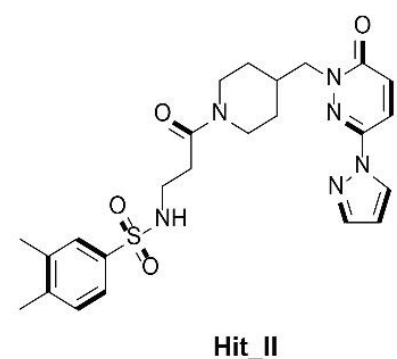

Hit_I

L

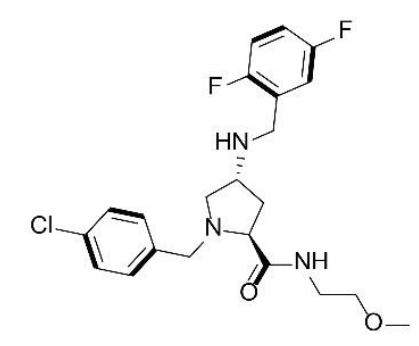

Hit_III

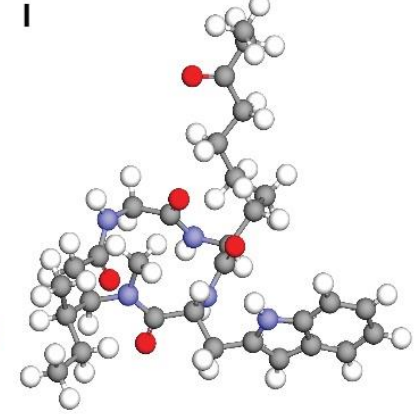

Hit_IV

Figure 3. Pharmacophore modeling and 2D-3D molecular structures of identified four hits. (A) Crystal structure of PICK1 PDZ domain with BQA inhibitor (PDB ID: 6AR4). (B) 2D molecular structure of BQA. Ocean blue dashed circles indicate the three hydrophobic pockets, R1, R2, and R3 in BQA. (C) 3D molecular structure of pharmacophore surrounded with the interacting amino acid residues of PDZ domain. (D) Pharmacophore model constructed at Pharmit web server. (E) 3D spatial distribution of the seven pharmacophore features. Distances between features; (1) F1-F2: $2.78 \AA$ (2) F1-F3:9.03 $\mathrm{\text {(3) } F 1 - F 4 : ~ 7 . 5 0 ~} \AA$ (4) F1-F5: $7.20 \AA$ (5) F1-F6: $8.78 \AA$ (6) F1-F7: $11.85 \AA$ (7) F2-F3: 7.33 $\AA$ (8) F2-F4: $6.85 \AA$ (9) F2-F5: $6.48 \AA$ (10) F2-F6: 9.31 A (11) F2-F7: $12.58 \AA$ (12) F3-F4: $3.70 \AA$ (13) F3-F5: $4.83 \AA$ (14) F3-F6: 7.57 $\AA$ (15) F3-F7: $10.01 \AA$ (16) F4-F5: $2.24 \AA$ (17) F4-F6: 4.00 $\AA$ (18) F4-F7: $6.90 \AA$ (19) F5-F6: $4.55 \AA$ (20) F5-F7: 7.64 A, and (21) F6-F7: $3.23 \AA$. (F-I) 3D molecular structures of obtained four hits, Hit_I (CHEMBL232154), Hit_II (KWFSWQGHHVLWDM-UHFFFAOYSA-N), Hit_III (MolPort-005-050-255), and Hit_IV (PubChem-46907406) respectively. (J-M) 2D molecular structures of obtained four hits, Hit_I (CHEMBL232154), Hit_II (KWFSWQGHHVLWDMUHFFFAOYSA-N), Hit_III (MolPort-005-050-255), and Hit_IV (PubChem-46907406) respectively.

Pharmacophore was constructed from the previously reported crystal structure of PICK1 PDZ domain with a small organic molecule inhibitor (BQA). BQA has total of 13 features, aromatic-2 (1. phenyl ring $\left[R-\underline{\mathrm{C}}_{6} \mathrm{H}_{4} \mathrm{CF}_{3}\right]$ and 2. phenyl ring $\left[\mathrm{R}-\underline{\mathrm{C}}_{6} \mathrm{H}_{4} \mathrm{Br}\right]$ ), hydrogen donor-1 (amide group nitrogen [R-CONH-R]), hydrogen acceptor-4 (1. amide group oxygen [R-CONH-R], 2. hydroxyl oxygen in the carboxylic acid group $[\mathrm{R}-\mathrm{C}=\mathrm{O}(\underline{\mathrm{O}} \mathrm{H})]$, 3. carbonyl oxygen in the carboxylic acid group $[\mathrm{R}-\mathrm{C}=\underline{\mathrm{O}}(\mathrm{OH})]$, and 4. piperidine nitrogen $\left[\mathrm{R}_{2}-\mathrm{C}_{5} \mathrm{H}_{8} \underline{\mathrm{N}}-\mathrm{R}\right]$ ), hydrophobic-5 (1. phenyl ring $\left[\mathrm{R}-\underline{\mathrm{C}_{6}} \mathrm{H}_{4} \mathrm{CF}_{3}\right]$, 2. phenyl ring $\left[\mathrm{R}-\mathrm{C}_{6} \mathrm{H}_{4} \mathrm{Br}\right], 3$. bromine atom $\left[\mathrm{R}-\mathrm{C}_{6} \mathrm{H}_{4} \mathrm{Br}\right]$, 4. trifluoromethyl group $\left[\mathrm{R}-\mathrm{C}_{6} \mathrm{H}_{4} \mathrm{CF}_{3}\right]$, and 5. cyclopropyl ring $\left.\left[\mathrm{R}-\underline{\mathrm{CH}}-\left(\mathrm{CH}_{2}\right)_{2}\right]\right)$, and negative ion-1 (carboxylic acid group [R- $\underline{\mathrm{COO}}$ ]) but, BQA interacts with PDZ through nine features (aromatic-1, hydrogen donor-1, hydrogen acceptor-3, hydrophobic-4) excluding four features (1. aromatic- phenyl ring $\left[R-\underline{\mathrm{C}}_{6} \underline{\mathrm{H}}_{4} \mathrm{Br}\right]$, 2. hydrogen acceptor- piperidine nitrogen $\left[\mathrm{R}_{2}-\mathrm{C}_{5} \mathrm{H}_{8} \underline{\mathrm{N}}-\mathrm{R}\right]$, 3 . 
hydrophobic- phenyl ring [R- $\left.\underline{\mathrm{C}} 6 \underline{\mathrm{H}}_{4} \mathrm{CF}_{3}\right]$, and 4. negative ion- carboxylic acid group [R- $\left.\underline{\mathrm{COO}}\right]$ ]). However, in pharmacophore construction, seven out of total nine were considered excluding two features (1. hydrophobic- cyclopropyl ring $\left[\mathrm{R}-\mathrm{CH}-\left(\mathrm{CH}_{2}\right)_{2}\right]$ and 2. hydrogen acceptor- carboxylic acid group's hydroxyl oxygen $[\mathrm{R}-\mathrm{C}=\mathrm{O}(\underline{\mathrm{OH}})]$ ). Figure $3 \mathrm{~A}-\mathrm{E}$ represents the $\mathrm{PDZ}$ domain structure with $\mathrm{BQA}$ and pharmacophore features.

There were ten molecular libraries (Table 1) screened against the PICK1 PDZ domain and at the end, four hits (CHEMBL25-1, MCULE-ULTIMATE-1, MolPort-1, and PubChem-1) were identified (Table 2). These hits along with BQA (control) were further prepared for molecular docking study with the PDZ domain. Pharmacophore models and the structures of identified hits are shown in Figure 3.

\section{Molecular docking}

The binding affinity and interactions of four identified hits with the PICK1 PDZ domain were probed using the flexible molecular docking DockThor web server. Additionally, the control (BQA) was also docked with the PDZ domain. The 2D and 3D molecular structures of the four hits are shown in Figure 3F-M. DockThor calculates the total energy, intermolecular interaction energy, van der Waals energy, and electrostatic energy along with docking score. Intermolecular energy is the sum of van der Waals and electrostatic energy. Total energy comprises three terms, (1) intermolecular energy between the protein and ligand atom pairs, (2) intramolecular energy of 1-4 non-bonded atom pairs in ligand, and (3) torsional energy of ligand.

Table 2. Docking results of hits and control (BQA) with the PICK1 PDZ domain calculated at DockThor web server.

\begin{tabular}{|c|c|c|c|c|c|c|c|c|}
\hline Name & ID & $\begin{array}{c}\text { Molecular } \\
\text { Formula }\end{array}$ & $\begin{array}{c}\text { Molecular } \\
\text { Weight } \\
(\mathrm{g} / \mathrm{mol})\end{array}$ & $\begin{array}{c}\text { Docking } \\
\text { score } \\
(\mathrm{kcal} / \mathrm{mol})\end{array}$ & $\begin{array}{c}\text { Total } \\
\text { energy } \\
(\mathrm{kcal} / \mathrm{mol})\end{array}$ & $\begin{array}{c}\text { Intermolecular } \\
\text { interaction } \\
\text { energy } \\
(\mathrm{kcal} / \mathrm{mol})\end{array}$ & $\begin{array}{c}\text { vdW } \\
\text { energy } \\
\text { (kcal/ } \\
\text { mol) }\end{array}$ & $\begin{array}{c}\text { Electrostatic } \\
\text { energy } \\
(\mathrm{kcal} / \mathrm{mol})\end{array}$ \\
\hline Hit_I & CHEMBL232154 & $\mathrm{C}_{29} \mathrm{H}_{40} \mathrm{CIN}_{7} \mathrm{O}_{4}$ & 586.12 & -9.0 & 13.4 & -28.8 & -26.1 & -2.7 \\
\hline \multirow[t]{2}{*}{ Hit_II } & KWFSWQGHHVLWDM & & & & & & & \\
\hline & -UHFFFAOYSA-N & $\mathrm{C}_{24} \mathrm{H}_{30} \mathrm{~N}_{6} \mathrm{O}_{4} \mathrm{~S}$ & 498.60 & -8.2 & 3.5 & -35.3 & -22.5 & -12.9 \\
\hline Hit_III & MolPort-005-050-255 & $\mathrm{C}_{22} \mathrm{H}_{26} \mathrm{ClF}_{2} \mathrm{~N}_{3} \mathrm{O}_{2}$ & 437.91 & -8.9 & 38.1 & -36.6 & -21.0 & -15.6 \\
\hline Hit_IV & PubChem-46907406 & $\mathrm{C}_{31} \mathrm{H}_{45} \mathrm{~N}_{5} \mathrm{O}_{5}$ & 567.72 & -9.2 & 20.6 & -30.1 & -26.7 & -3.4 \\
\hline Control & BQA & $\mathrm{C}_{26} \mathrm{H}_{29} \mathrm{BrF}_{3} \mathrm{~N}_{2} \mathrm{O}_{3}$ & 554.419 & -8.6 & 16.9 & -49.7 & -21.6 & -28.1 \\
\hline
\end{tabular}

Total energy indicates the rank of different binding poses of the same ligand whereas docking score differentiates the ligands based on their interaction energy with a specific protein.

Docking score was found approximately in the range of -8.0 to $-9.0 \mathrm{kcal} / \mathrm{mol}$ (Table 2). From the results, it was noticed that three hits have slightly higher docking scores compared to the BQA while the remaining has slightly less than the BQA docking score. Among all, Hit_IV has the highest docking score $(-9.2 \mathrm{kcal} / \mathrm{mol})$ and Hit_ll has the lowest $(-8.2 \mathrm{kcal} / \mathrm{mol})$. The 3D surface 
representations (Figure 4A-D: H-bond and Figure 4E-H: hydrophobic) and the 2D interactions (Figure 4I-L) of studied hits with the PDZ domain are illustrated in Figure 4.

Hit_I (CHEMBL232154) derivatives have been reported as motilin receptor inhibitors (Marsault et al., 2007). Docking score of Hit_I with PDZ domain was observed $-9.0 \mathrm{kcal} / \mathrm{mol}$. In the Hit_IPDZ complex, Lys83 forms a hydrogen bond with carbonyl oxygen, and Phe53 interacts with the aromatic ring through pi-pi stacking. However, mainly hydrophobic interactions (pi-alkyl and alkyl-alkyl) were observed with the Leu32, Ile37, and Ala87 amino acids (Figure 4l). Additionally, the chlorine atom interacts with the Leu32 and contributes to the total interaction energy. Lys83 and Ala87 are the part of $\alpha$-helix while Ile37 is present at the $\beta$-strand region. The second compound Hit_Il, has the lowest docking score $(-8.2 \mathrm{kcal} / \mathrm{mol})$ among all the compounds. Through the literature review, we found that this compound has never been reported as an inhibitor for any target. There were three hydrogen bonds observed between the compound and surrounding residues (lle35, Ile37, and Gln91). Three heterocyclic moieties, pyridazine, piperidine, and pyrazole interact with the Val84, Ala87, and Lys88 $\alpha$-helix amino acids respectively (Figure $4 \mathrm{~J}$ ). However, the remaining dialkyl substituted aromatic ring has hydrophobic interactions with Leu32 and Phe53 residues which are present at the loop region of the PDZ domain.

Next, the Hit_III was also observed to have a good docking score $(-8.9 \mathrm{kcal} / \mathrm{mol})$ towards the PDZ domain. From the interactions shown in Figure $4 \mathrm{~K}$, it can be assumed that multiple hydrophobic interactions of two halogenated aromatic rings with Lys27, Leu32, Ile33, Ile90, and Phe53 vicinal amino acid residues contribute significantly to the total binding energy of the compound with PDZ domain.

In addition to this, it also forms two hydrogen bonds with Ser36 and lle37 through oxygen atoms of ether and amide functional groups respectively. Only a single amino acid (lle90) is located in $\alpha$-helix while remaining residues come from the $\beta$-strand and loop regions of the PDZ domain. This compound has also not been reported previously in the literature as an antagonist for any protein target. Lastly, Hit_IV showed the highest binding $(-9.2 \mathrm{kcal} / \mathrm{mol})$ with the PDZ domain. 
A

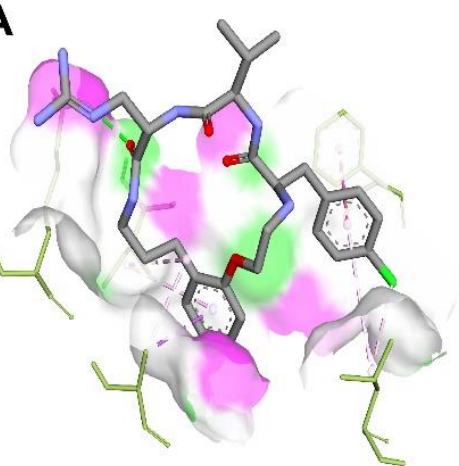

B

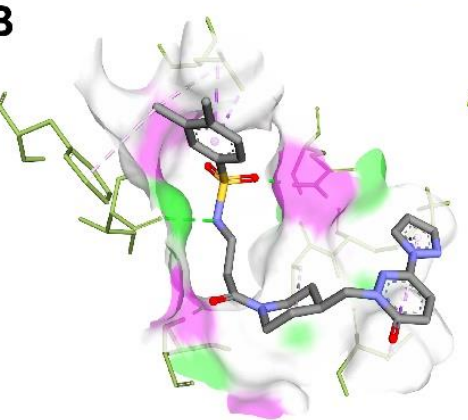

C

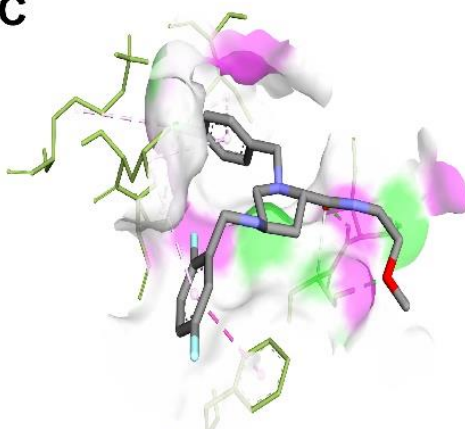

D

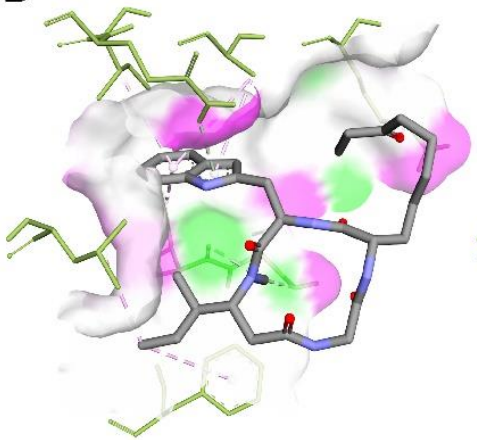

E

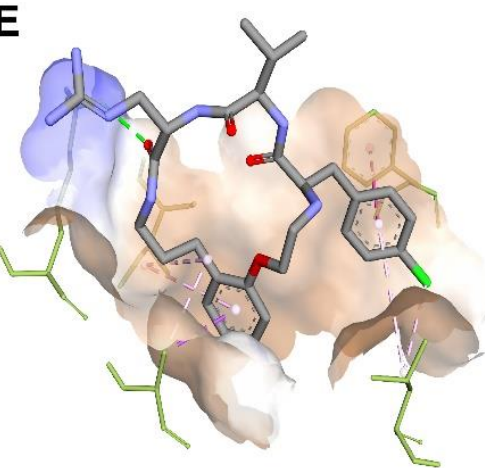

F

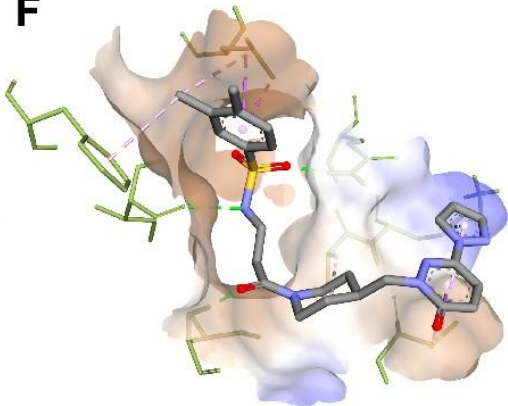

G

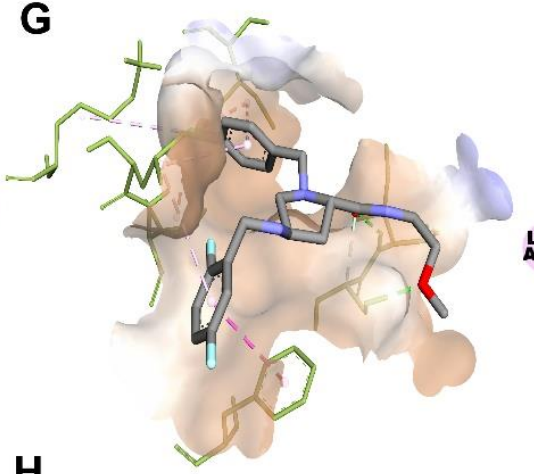

H

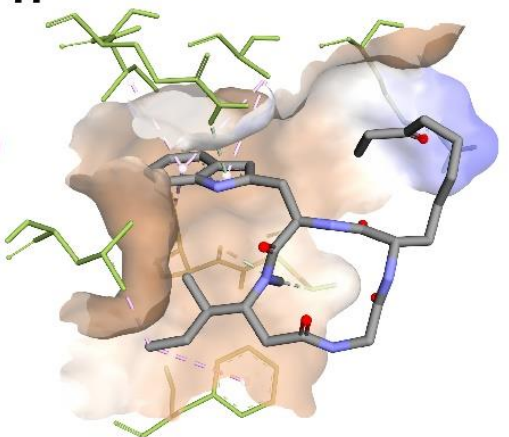

I

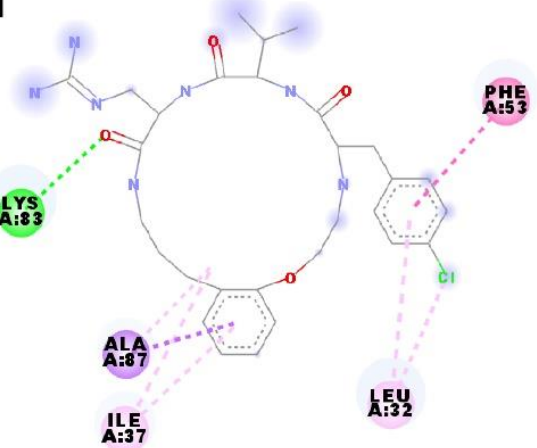

J

개곤

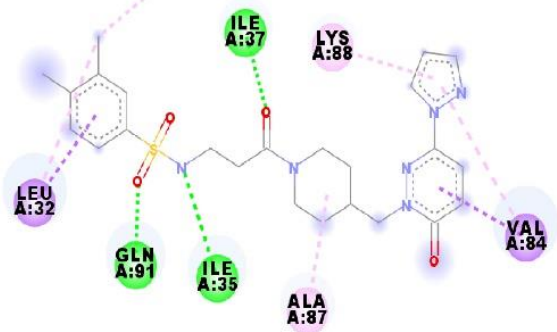

K

ALE

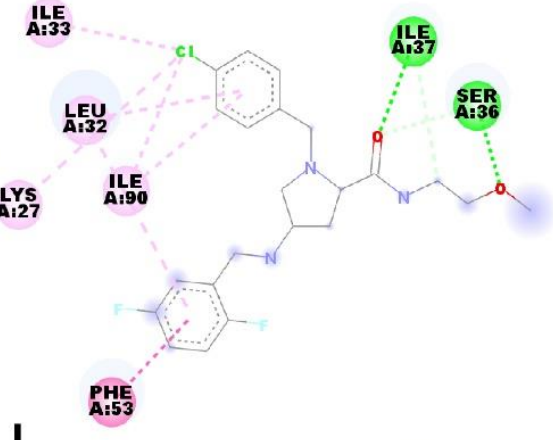

L

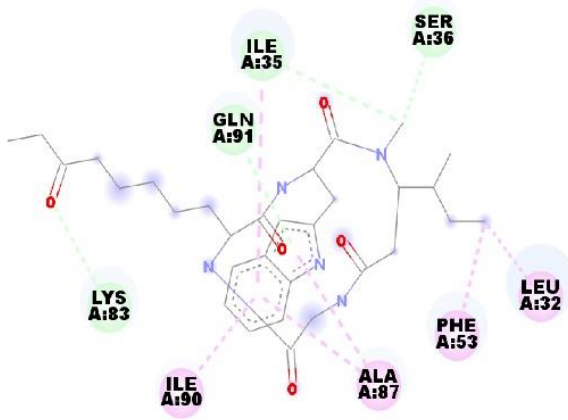

\section{H-Bonds \\ Donor}

\section{Hydrophobicity}

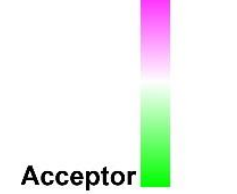

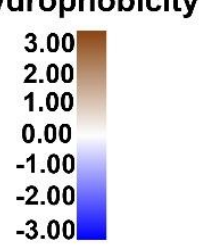

Conventional hydrogen bond Pi-Sigma Pi-Pi stacked

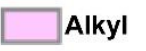

Pi-Alkyl

Figure 4. 2D and 3D Interactions between the hits and PDZ domain. (A-D) Hydrogen bond surface representations of Hit I to Hit_IV respectively. (E-H) Hydrophobic surface representations of Hit_I to Hit_IV respectively, and (I-L) 2D interactions of Hit_I to Hit_IV respectively.

Like other hits, this compound also has multiple hydrophobic interactions with the proximal amino acids such as Leu32, Ile35, Phe53, Ala87, and Ile90 in the binding pocket (Figure 4L). 
The indole aromatic ring interacts with Gln91 through the pi-donor hydrogen bond along with other hydrophobic interactions. Furthermore, Ile35 and Ser36 residues of $\beta$-strand form slightly polar carbon-hydrogen bonds with the amide group hydrogen and Lys 83 from the $\alpha$-helix interacts through its terminal $-\mathrm{CH} 2$ - hydrogen with the ketonic oxygen atom of hydrophobic alkane chain in the compound.

Lifeng Pan and his team (Pan et al., 2007) have solved the crystal structure of the PICK1 PDZ domain with GluA1 tail peptide and indicated which residues of the PDZ domain are responsible for PZD-GluA2 interaction for the internalization of AMPA receptor. Their interactions analysis suggests that Lys27, Ile33, Gly34, Ile35, Ser36, Ile37, Lys83, Ala87, and Ile90 amino acids significantly interact with the GluA2 peptide tail and are responsible for facilitating the AMPA internalization. Isoleucine plays the dominant role to bind GluA2 peptide. To understand the allosteric mechanism of Hit_III, GluA2 peptide was docked with the PDZ-Hit_III from the 7 ns frame of previous simulation and docking score was observed $-19.8 \mathrm{kcal} / \mathrm{mol}$. Further, Hit_III was removed to probe the dynamics of GluA2 peptide alone inside the PDZ binding pocket and molecular dynamics was performed for the two complexes, PDZ-GluA2 and PDZ-GluA2-Hit_III.

\section{Molecular dynamics simulation}

\section{Root mean square deviation (RMSD)}

To get insight into the dynamicity of protein-ligand complexes, 50 ns all-atom molecular dynamics simulation was performed using the GROMACS tool. The protein conformational changes and secondary structure stability upon ligand binding were investigated by analyzing 50 ns MD trajectories for Root mean square deviation (RMSD) of the protein backbone and Root mean square fluctuation (RMSF) of alpha-carbon atoms of the protein. Figure 5A represents the RMSD plot. Among all the complexes, the mean RMSD of PDZ-BQA was observed highest $(1.0 \mathrm{~nm})$. PDZ-Hit_ll also showed significant RMSD $(0.94 \mathrm{~nm})$ while the remaining had an average $0.75 \mathrm{~nm}$ RMSD. RMSD of ligand unbound PDZ was noticed $0.67 \mathrm{~nm}$ which indicates that upon ligand binding, protein dynamicity increases significantly. Additionally, it was observed that for the initial 20 ns of simulation, the RMSD of all the complexes except PDZ-BQA were increased and then remained constant between $0.5 \mathrm{~nm}-1.2$ $\mathrm{nm}$. Hence, ligand binding PDZ gains stability for the remaining simulation time. Also, tested hits give more stability to the PDZ domain in comparison with control BQA.

Furthermore, the RMSD of each ligand was also computed from the 50 ns trajectory of each complex. Figure 5B illustrates the RMSD of four hits along with BQA. It can be seen from Figure 5B that Hit_II showed the highest mean RMSD $(2.2 \mathrm{~nm})$ which indicates its higher dynamicity inside the protein. Hit_III fluctuates considerably for the first 7-8 ns and then it remains stable during the last $42 \mathrm{~ns}$. Hit_IV also changes its conformations significantly (mean RMSD: 0.93 $\mathrm{nm}$ ) upon protein binding during the simulation compared to the remaining ones (mean RMSD: $0.55 \mathrm{~nm})$. 

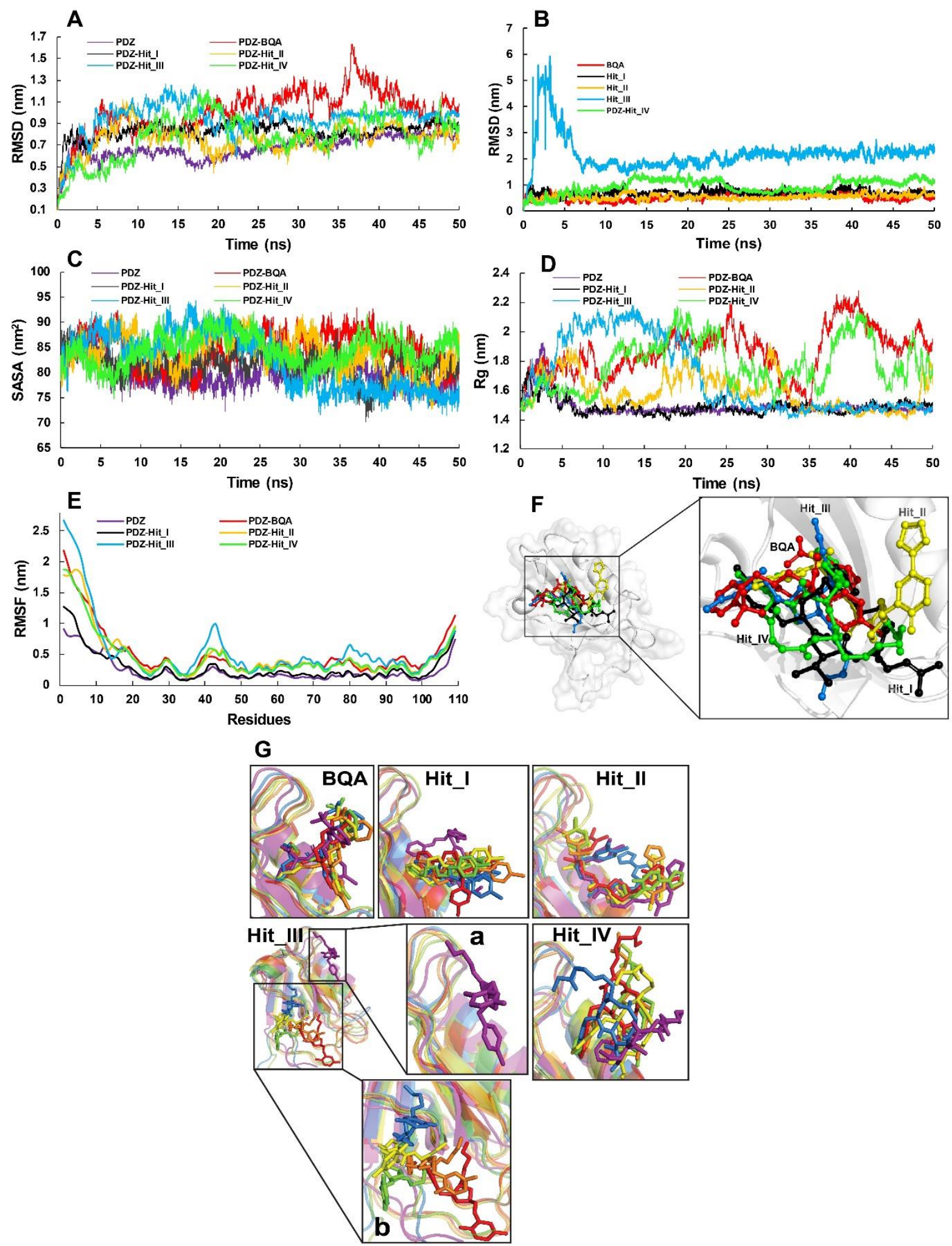

Figure 5. MD simulations analysis of hits and control (BQA) with the PDZ domain and their docked poses at the PDZ binding pocket. (A) RMSD (nm) plots of the PDZ backbone with the ligand bound and unbound states. (B) RMSD (nm) plots of the ligands. (C) SASA $\left(\mathrm{nm}^{2}\right)$ plots of PDZ with ligand bound and unbound states. (D) $\mathrm{Rg}(\mathrm{nm})$ plots of PDZ with ligand bound and unbound states. (E) RMSF $(\mathrm{nm})$ plots of C-alpha atoms in PDZ with ligand bound and unbound states, and (F) Docked poses of four hits and control (BQA) inside the PDZ catalytic pocket. (G) Aligned ligand poses from the MD trajectories at every $10 \mathrm{~ns}$. 


\section{Solvent accessible surface area (SASA)}

To further understand the PDZ domain stability and conformational changes upon ligand binding, Solvent accessible surface area (SASA) was determined from the 50 ns trajectory for all the complexes along with PDZ alone. The mean values were observed $79.9 \mathrm{~nm}^{2}, 84.7 \mathrm{~nm}^{2}$, $81.7 \mathrm{~nm}^{2}, 83.8 \mathrm{~nm}^{2}, 82.2 \mathrm{~nm}^{2}$, and $84.5 \mathrm{~nm}^{2}$ for PDZ, PDZ-BQA (control), PDZ-Hit_I, PDZ-Hit_II, PDZ-Hit_III, and PDZ-Hit_IV respectively. The high SASA value indicates the structure expansion which leads to increased solvent access of the surface area of protein. The mean SASA value does not give a clear indication of the influence of ligand binding on protein stability. Hence, trajectory with respect to simulation time was interpreted. From the trajectory analysis, it was observed that the SASA for the PDZ-Hit_III was reduced significantly compared to other complexes including control. Additionally, the SASA for the PDZ domain alone was also reduced at the end of the simulation. For the remaining ones, SASA was not significantly increased or decreased but fluctuated between time steps which indicates the slightly opening and closing of the PDZ domain upon ligand binding. Thus, the SASA value suggests that Hit_III efficiently stabilizes the PDZ domain upon its binding. Figure $5 \mathrm{C}$ represents the SASA plots.

\section{Radius of gyration ( $\mathbf{R g})$}

Protein compactness variation during the simulation can be measured by calculating the Radius of gyration (Rg) of protein. The mean values of Rg of PDZ, PDZ-BQA, PDZ-Hit_I, PDZ-Hit_II, PDZ-Hit_III, and PDZ-Hit_IV were observed at $1.5 \mathrm{~nm}, 1.9 \mathrm{~nm}, 1.5 \mathrm{~nm}, 1.6 \mathrm{~nm}, 1.7 \mathrm{~nm}$, and 1.8 $\mathrm{nm}$ respectively. There was a significant deviation in compactness observed for all the complexes except PDZ and PDZ-Hit_I (Figure 5D). Rg values of PDZ and PDZ-Hit_I complex were increased during the first $2 \mathrm{~ns}$ simulation. Then, they remained constant between 1.4-1.5 $\mathrm{nm}$. However, in the case of PDZ-Hit_III, Rg was observed between 1.5-2.2 nm in the initial 20 $\mathrm{ns}$, and then, it was quite stable $(1.4-1.5 \mathrm{~nm})$ during the remaining simulation. For PDZ-Hit_II, Rg was observed considerably less (1.4-1.9 nm) than the Rg values (1.5-2.3 nm) of PDZ-Hit_IV and PDZ-BQA. Thus, PDZ-Hit_I, PDZ-Hit_II, and PDZ-Hit_III complexes become compact at the end of the simulation which indicates the stabilization of PDZ through ligand binding. Figure $5 F$ shows the docked poses of hits and BQA with PDZ domain.

\section{Root mean square fluctuation (RMSF)}

RMSF analysis indicates how protein residues fluctuate with the ligand binding. Figure $5 \mathrm{E}$ illustrates the RMSF plot. Leaving C- and N-terminal regions (C-terminal: 101-109, N-terminal: 1-20), average RMSF values of PDZ alone and ligand-bound complexes were computed. RMSF values indicate that the PDZ unbound state has the lowest RMSF $(0.15 \mathrm{~nm})$ whereas PZDHit_III has the highest fluctuations (RMSF: $0.38 \mathrm{~nm}$ ). In the other complexes, mean RMSF was observed $0.28 \mathrm{~nm}$. Furthermore, interestingly it was observed that ligand binding residues (Lys27, Leu32, Ile33, Ile35, Ser36, Ile37, Phe53, Lys83, Val84, Ala87, Lys88, Ile90, and GIn91) do not fluctuate significantly. However, the residues, Gly40, Ala41, Gln42, Tyr43, Cys44, Pro45, and Cys46 in the loop region connecting two beta-strands and residues, Lys79, Gly80, and Thr81 in another loop connecting helix and beta-strand showed higher fluctuations. 
To probe the structural deviation of the hits and BQA, $1 \mathrm{~ns}, 10 \mathrm{~ns}, 20 \mathrm{~ns}, 30 \mathrm{~ns}, 40 \mathrm{~ns}$, and 50 ns frames from each trajectory were extracted and superimposed in PyMOL. Figure 5G illustrates the superimposed complexes of hits and BQA. It can be seen from the Figure $5 G$ that Hit_I, Hit_II, and BQA show less deviation while Hit_III (Allosteric pocket) and Hit_IV deviate significantly. The average RMSD values of BQA, Hit_I, Hit_II, Hit_III, and Hit_IV were observed $3.1 \AA$, $6.0 \AA$, $3.4 \AA$, $7.1 \AA$, and $7.4 \AA$ respectively. Thus, Hit_ll shows the lowest RMSD compared to other hits and consequently, it can be considered best hit for the PDZ domain.

\section{Hydrogen bonding}

Polar interactions between the protein and ligand contribute significantly to the binding affinity between them. Conventional hydrogen bond formation between the protein and ligand stabilizes the complex. Apart from RMSD, RMSF, SASA, and Rg, intramolecular hydrogen bond formation in PDZ domain and intermolecular hydrogen bond formation between the ligand and protein were calculated from the 50 ns trajectory. Intramolecular hydrogen bonds in PDZ and intermolecular hydrogen bonds in complexes are depicted in Figure 6A and Figure 6B-F respectively. The average number of intramolecular hydrogen bonds in PDZ, PDZ-BQA, PDZHit_I, PDZ-Hit_II, PDZ-Hit_III, and PDZ-Hit_IV were identified 55, 52, 54, 52, 54, and 50 respectively. Thus, Hit_IV disrupts the intramolecular hydrogen bond network in PDZ to a greater extent compared to other hits and control BQA. Hit_II, Hit_IV, and BQA form a maximum of five intermolecular hydrogen bonds with the protein compared to Hit_I and Hit_III which form three and two respectively. During the $50 \mathrm{~ns}$ simulation, hydrogen bonds between the protein and ligand were persistent in all the complexes except PDZ-Hit_III. Hence, hydrogen bonding contributes significantly to the binding energy of all complexes except PDZ-Hit_III. 

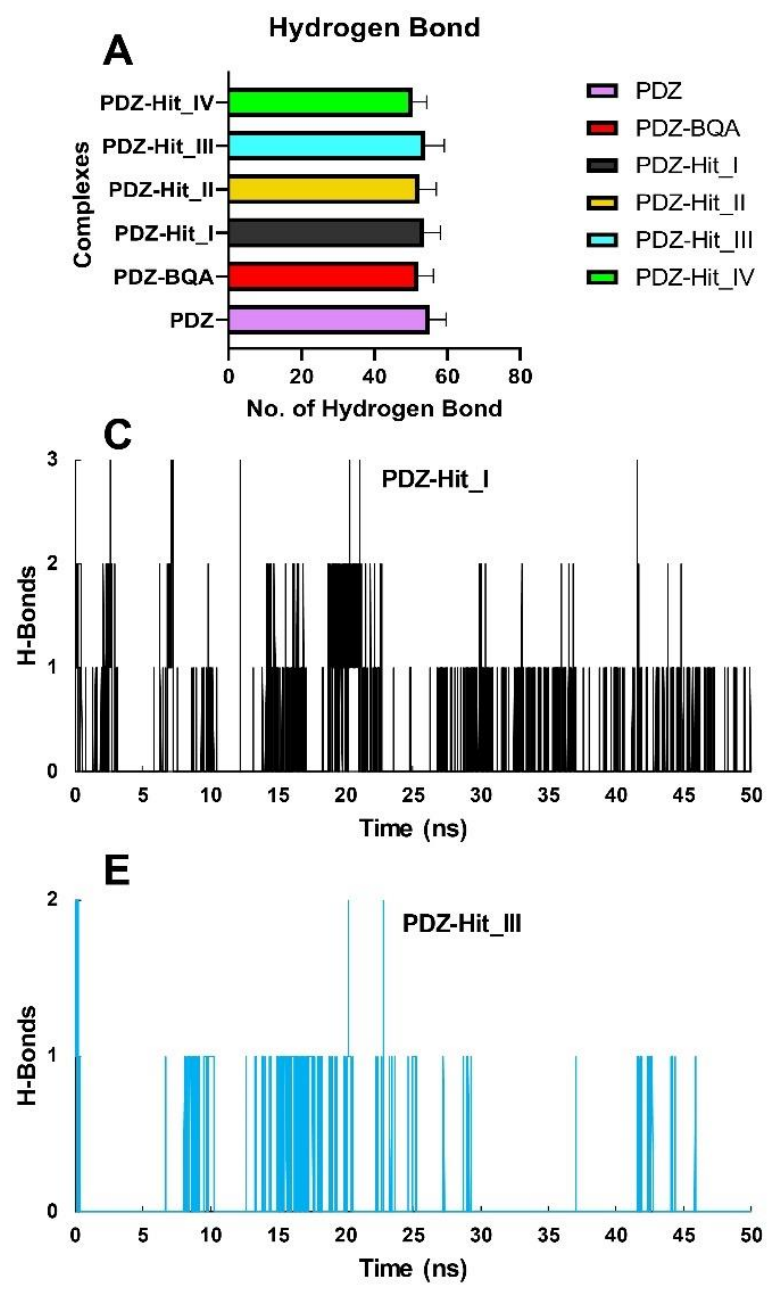
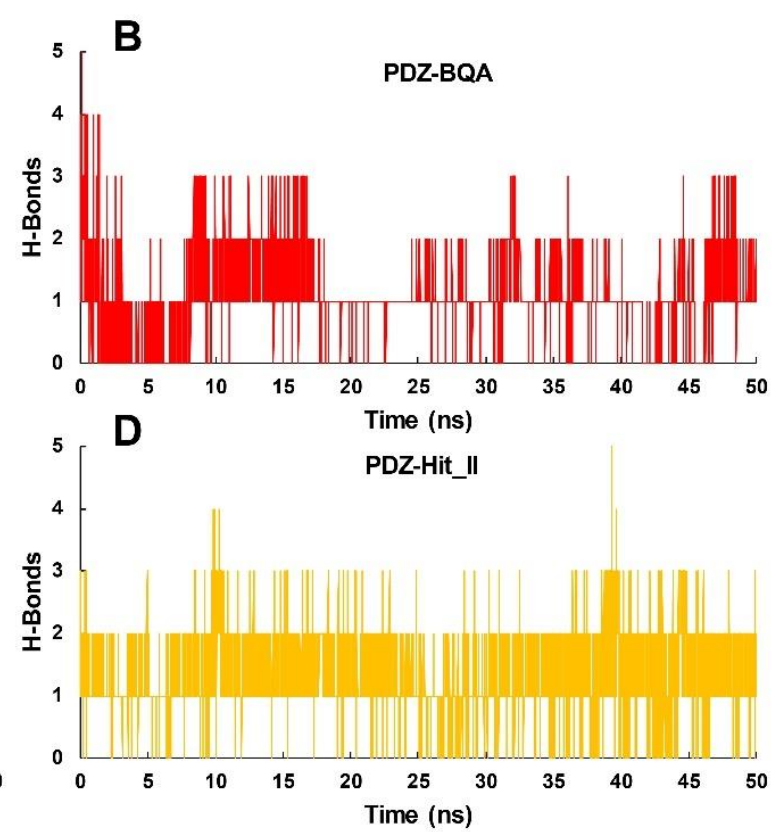

$\mathbf{F}$

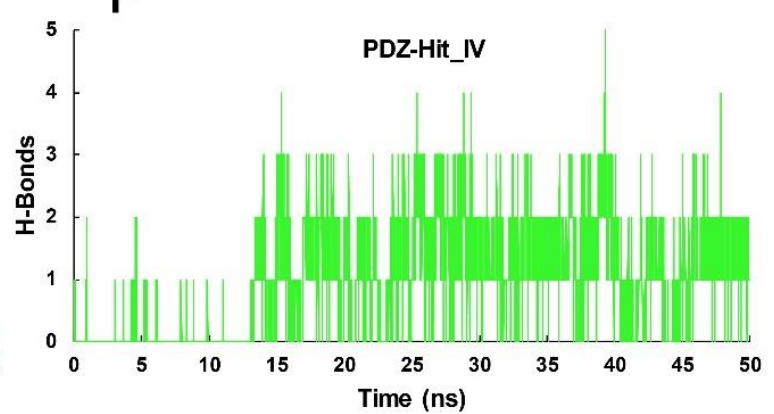

Figure 6. MD simulation analysis of Intramolecular and intermolecular hydrogen bonds in PDZ and its complexes. (A) Intramolecular hydrogen bonds in PDZ with ligand bound and unbound states and, (B-F) Intermolecular hydrogen bonds in the complexes of PDZ with the Hit_I to Hit_IV respectively.

\section{Allosteric inhibition}

As it can be seen from the Figure 5B, Hit_III shows big jump during initial 5-7 ns and then, its RMSD remains in equilibrium for the rest of the simulation. To scrutinise this abnormal behavior, we carefully visualized the simulation and observed that Hit_III dissociates from the active site (52.9 $, 46.2 \AA, 14.0$ $\AA$ A) and reassociates with another pocket on the PDZ domain. Figure 7 illustrates the allosteric mechanism of Hit_III. Figure 7A shows the PDZ-Hit_III complex dynamics in which Hit_III is leaving the AMPA GluA2 binding pocket and searches for a new pocket (58.3 $\mathrm{A}, 41.4 \AA, 29.5 \AA$ ) that was found as an allosteric site in the later simulations. The residues, Tyr48, Ile49, Val50, Gly67, Gln106, and Ala108 are involved in allosteric binding pocket on PDZ domain.

To probe whether Hit_III behaves as an allosteric inhibitor, frame at the $7 \mathrm{~ns}$ from the previous simulation trajectory was extracted and the AMPA GluA2 peptide was docked with the complex (PDZHit_II) using peptide docking. Finally, two simulations (50 ns) for the PDZ-GluA2 and PDZ-GluA2-Hit_III 
complexes were performed. Interestingly, it was noted that Hit_III acts as an allosteric inhibitor. Figure 7B illustrates the allostery mechanism and, it was found that in absence of Hit_III, AMPA GluA2 peptide resides at its catalytic pocket (Figure 7C-I) however, in presence of Hit_III, it departs significantly from its actual binding position (Figure 7B-II). Thus, this observation proves the allosteric nature of Hit_III. Figure 7B-III represents the RMSDs of GluA2 peptide in presence and absence of Hit_III and RMSD of Hit_III in presence of GluA2 peptide. The average RMSD of peptide alone was observed $0.8 \mathrm{~nm}$ and shows equilibrium during whole simulation. Nevertheless, GluA2 fluctuates dramatically (average RMSD: $3.5 \mathrm{~nm}$ ) in presence of Hit_III during the simulation. The average RMSD of Hit_Ill was observed $1.7 \mathrm{~nm}$ which shows slight deviation from its initial position and then remains stable. The behavior of PDZ domain in the PDZ-GluA2 and PDZ-GluA2-Hit_III complexes was also analysed (Figure 7C-F) using the RMSD, SASA, Rg, and RMSF analyses. The RMSD of PDZ domain in PDZ-GluA2-Hit_III complex was observed slightly more in comparison with complex PDZ-GluA2. After $40 \mathrm{~ns}$, RMSD remained constant around at $0.35 \mathrm{~nm}$ in PDZ-GluA2 complex whereas, it was reduced to around 0.2 $\mathrm{nm}$ in PDZ-GluA2-Hit_III complex which indicates the slight conformational transition in PDZ domain.

The overall SASA value of PDZ domain in PDZ-GluA2-Hit_III complex was observed marginally less (average: $77.1 \mathrm{~nm}^{2}$ ) than the SASA (average:78.0 $\mathrm{nm}^{2}$ ) in PDZ-GluA2 complex. Additionally, the average $\mathrm{Rg}$ value of PDZ domain in both the complexes was noted around $1.5 \mathrm{~nm}$. For the first $18 \mathrm{~ns}$ in PDZ-GluA2-Hit_III, Rg was observed moderately less than the Rg in PDZ-GluA2 complex. Thus, SASA and Rg values suggest increased compactness of PDZ-GluA2-Hit_III complex. In both the complexes, three loop regions $(25-31,40-45,52-60)$ near the active site showed more fluctuation (Average RMSF, 25-31: $0.18 \mathrm{~nm}, 40-45: 0.25 \mathrm{~nm}, 52-60: 0.22 \mathrm{~nm}$ ). Thus, above results confirm the allosteric nature of Hit_II with the PDZ domain and GluA2 peptide binding. 

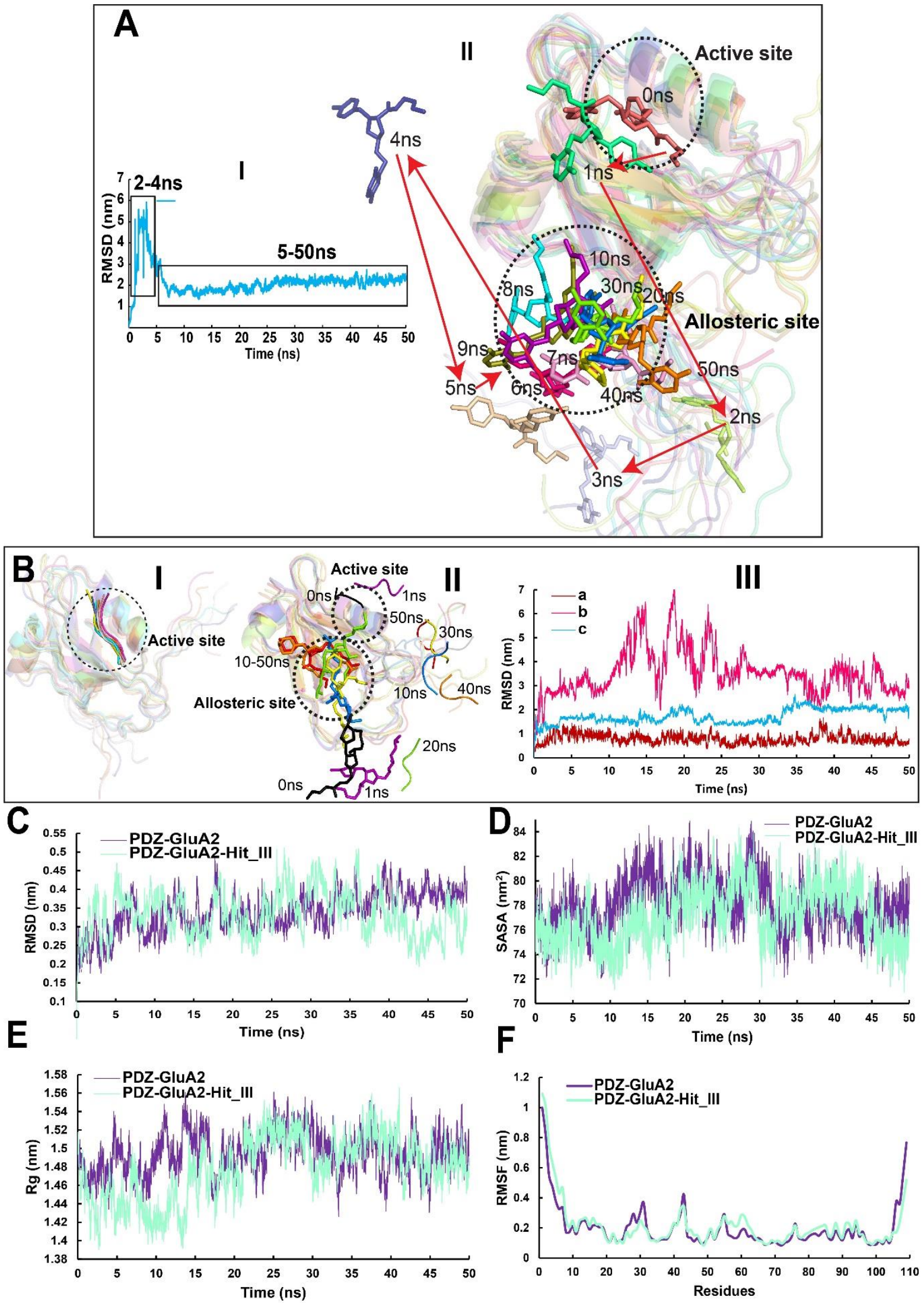

Figure 7. (A) Deviation of Hit_III binding from the active site. A-I shows the Hit_III RMSD plot where, during the first 24 ns simulation, it stays at the active site then finds other binding pocket (allosteric site) and stays there for rest of the simulation. A-II illustrates the Hit_III path while changing its binding sites. (B) Allosteric mechanism of Hit_III. B-I represent the behavior of AMPA GluA2 peptide without Hit_III during the 50 ns simulation. B-II illustrates the how Hit_III 
allosterically impacts the binding of AMPA GluA2 peptide from the active site. B-III shows the RMSD of AMPA GluA2 peptide alone (III-a), AMPA GluA2 peptide in presence of Hit_III (III-b), and Hit_III in presence of GluA2 peptide (III-c). (C-F) RMSD, SASA, Rg, and RMSF of PDZ domain respectively. Purple color indicates the data for the PDZ domain in PDZ-GluA2 complex while cyan, in PDZ-GluA2- Hit_III complex.

\section{ADME analysis}

To investigate the ADME properties of four hits and control, physiochemical parameters, lipophilicity, water solubility, pharmacokinetics, and drug-likeness characteristics were analyzed using the widely used SwissADME web server.

\section{Lipinski's rule of five (RO5) and bioavailability scores}

For the drug-likeness, all the hits were passed through Lipinski's rule of five (RO5). Small organic molecules are classified using these rules based on their physicochemical properties, which includes molecular weight $(\mathrm{MW})<500 \mathrm{~g} / \mathrm{mol}$, MLOGP $\leq 4.15$, hydrogen bond acceptors $(\mathrm{N}$ or $\mathrm{O}) \leq 10$, hydrogen bond donors $(\mathrm{NH}$ or $\mathrm{OH}) \leq 5$, and molar refractivity between the 40 and 130 (Lipinski et al., 2001). For drug-likeness prediction, Hit_II and Hit_III obey Lipinski's rule of five with zero violation. Hit_IV and control (BQA) violate a single rule while Hit_I violates three rules (Table 3). In addition to this, bioavailability scores of hits and control were calculated using Abbot bioavailability score (ABS) method (Martin, 2005). According to this method, candidates must have at least 0.10 oral bioavailability in rat to show drug-likeness. For the Hit_l, score was predicted less $(0.17)$ while, for the remaining hits and control (BQA) score was observed high (0.55).

Table 3. SwissADME predicted physicochemical properties, lipophilicity, water solubility, pharmacokinetics, and drug-likeness of four hits and control (BQA).

\begin{tabular}{|c|c|c|c|c|c|c|}
\hline Parameters & Molecule & Hit_I & Hit_II & Hit_III & Hit_IV & Control (BQA) \\
\hline \multirow{8}{*}{$\begin{array}{l}\text { Physicochemical } \\
\text { Properties }\end{array}$} & Formula & $\mathrm{C}_{29} \mathrm{H}_{41} \mathrm{CIN}_{7} \mathrm{O}_{4}$ & $\mathrm{C}_{24} \mathrm{H}_{30} \mathrm{~N}_{6} \mathrm{O}_{4} \mathrm{~S}$ & $\mathrm{C}_{22} \mathrm{H}_{28} \mathrm{ClF}_{2} \mathrm{~N}_{3} \mathrm{O}_{2}$ & $\mathrm{C}_{31} \mathrm{H}_{45} \mathrm{~N}_{5} \mathrm{O}_{5}$ & $\mathrm{C}_{26} \mathrm{H}_{29} \mathrm{BrF}_{3} \mathrm{~N}_{2} \mathrm{O}_{3}$ \\
\hline & Molecular Weight (g/mol) & 587.13 & 498.6 & 439.93 & 567.72 & 554.42 \\
\hline & Fraction Csp3 & 0.45 & 0.42 & 0.41 & 0.58 & 0.46 \\
\hline & No. of Rotatable bonds & 5 & 9 & 10 & 11 & 10 \\
\hline & No. of H-bond acceptors & 5 & 7 & 4 & 5 & 6 \\
\hline & No. of $\mathrm{H}$-bond donors & 6 & 1 & 3 & 4 & 3 \\
\hline & Molar Refractivity & 174.92 & 142.85 & 117.89 & 174.87 & 134.48 \\
\hline & $\operatorname{TPSA}\left(\AA^{2}\right)$ & 177.54 & 125.35 & 59.38 & 140.47 & 70.84 \\
\hline \multirow{6}{*}{ Lipophilicity } & Log $\mathrm{P}_{\mathrm{o} / \mathrm{w}}$ (iLOGP) & 2.87 & 3.58 & 3.56 & 2.89 & 3.61 \\
\hline & Log $P_{o / w}(X L O G P 3)$ & 2.36 & 1.43 & 3.08 & 3.15 & 3.41 \\
\hline & Log $P_{o / w}(W L O G P)$ & -1.67 & 1.44 & 0.83 & 1.48 & 4.51 \\
\hline & $\log P_{o / w}(M L O G P)$ & -2.76 & 1.74 & -4.46 & 0.8 & 0.69 \\
\hline & Log $P_{o / w}($ Silicos-IT Log P) & 2.26 & 1.25 & 4.42 & 4.66 & 5.79 \\
\hline & Consensus Log $\mathrm{P}_{\mathrm{o} / \mathrm{w}}$ & 0.62 & 1.89 & 1.48 & 2.6 & 3.6 \\
\hline \multirow{4}{*}{ Water Solubility } & ESOL Log S & -4.85 & -3.47 & -4.14 & -4.78 & -5.02 \\
\hline & ESOL Solubility (mg/ml) & $8.22 \times 10^{-03}$ & $1.69 \times 10^{-01}$ & $3.16 \times 10^{-02}$ & $9.40 \times 10^{-03}$ & $5.30 \times 10^{-03}$ \\
\hline & ESOL Solubility (mol/l) & $1.40 \times 10^{-05}$ & $3.38 \times 10^{-04}$ & $7.18 \times 10^{-05}$ & $1.66 \times 10^{-05}$ & $9.56 \times 10^{-06}$ \\
\hline & ESOL Class & $\begin{array}{l}\text { Moderately } \\
\text { soluble }\end{array}$ & Soluble & $\begin{array}{l}\text { Moderately } \\
\text { soluble }\end{array}$ & $\begin{array}{l}\text { Moderately } \\
\text { soluble }\end{array}$ & $\begin{array}{l}\text { Moderately } \\
\text { soluble }\end{array}$ \\
\hline \multirow{2}{*}{ Pharmacokinetics } & Gl absorption & Low & High & High & High & High \\
\hline & BBB permeant & No & No & Yes & No & Yes \\
\hline
\end{tabular}




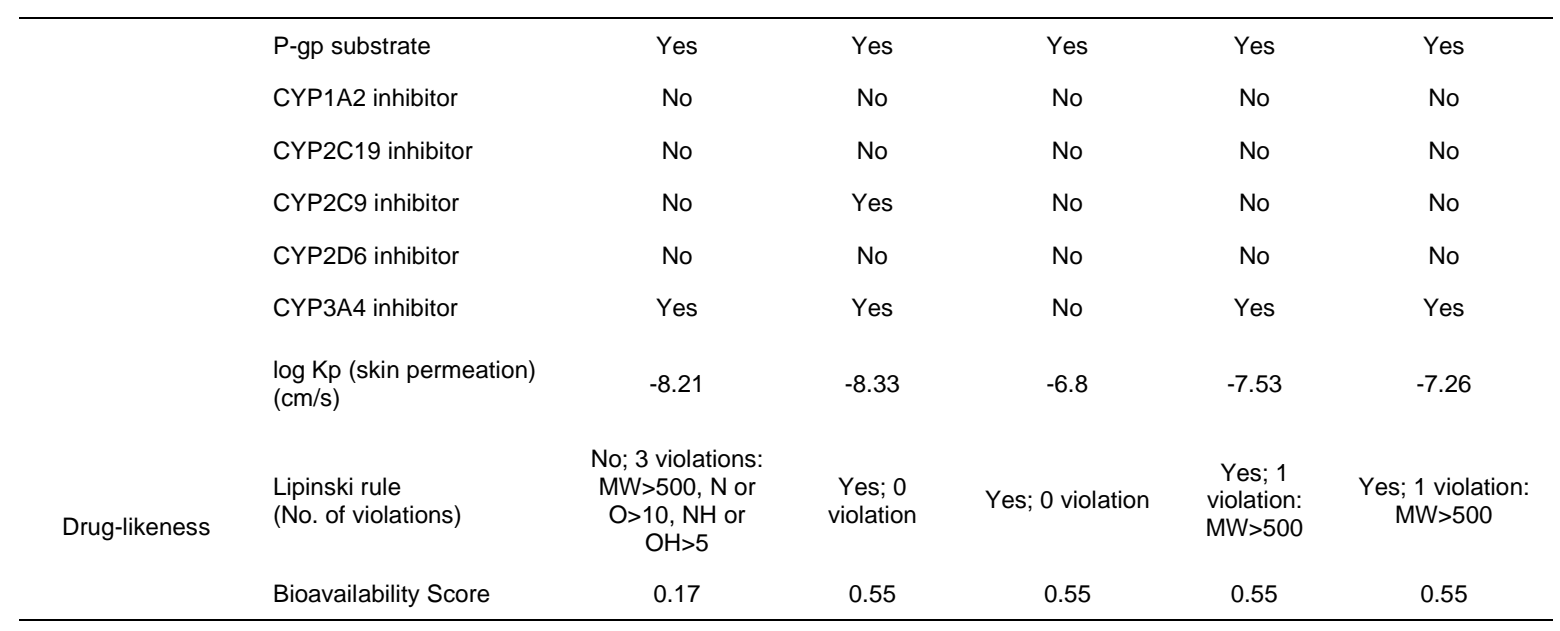

\section{Lipophilicity}

Lipophilicity indicates the solubility of compounds in oil or any nonpolar solvent (Johnson, 1998). To measure the lipophilicity, the partition coefficient between water and n-octanol is measured (Constantinescu et al., 2019). To compute such values, numerous ADME (Absorption, distribution, metabolism, and excretion) computational tools have been developed (Pires et al., 2015; Schyman et al., 2017; Yang et al., 2018) like SwissADME. SwissADME has five different lipophilicity (Log Po/w) prediction models such as XLOGP3 (Cheng et al., 2007), WLOGP (Wildman \& Crippen, 1999), MLOGP (Moriguchi et al.,1992), SILICOS-IT (http://silicos-it.be.s3-website-eu-west1.amazonaws.com/software/filter-it/1.0.2/filter-it.html, 2016), and iLOGP (Daina et al., 2014). Tested hits along with control (BQA) have the consensus lipophilicity values from 0.62 to 3.6 and which means these compounds follow the Lipinski rule of five $(\log P<5)$. Hence, these hits can be further validated for clinical trials (Arnott \& Planey, 2012). Hit_I has the lowest lipophilicity $(+0.62)$ whereas Hit_II and control have the higher values of lipophilicity, 2.6 and 3.6 respectively.

\section{Water solubility}

Water solubility of drugs is an important factor in drug design and development. It is used to measure drug concentration with an expected pharmacological response (Savjani et al., 2012). Poor solubility of compounds leads to less effective results and it is a great challenge in drug discovery. Solubility increases the drug concentration in the blood which is useful for its therapeutic effectiveness (Bergström \& Larsson, 2018). To determine the water solubility of hits, we used ESOL (Estimated solubility) model from the SwissADME (Delaney, 2004). Results show that Hit_II has the highest solubility (-3.47) while remaining hits and control (BQA) are predicted to be moderately soluble. The magnitude of Log $S$ was found in the range of -3.47 to -5.02 .

\section{Pharmacokinetics}

The pharmacokinetic properties of drugs involve drug Absorption, distribution, metabolism, and excretion (ADME). Pharmacokinetic characteristics are a significant factor for the drug selection against the particular therapeutic target (Jang et al., 2001). The high value of Gastrointestinal (Gl) absorption (Daina \& Zoete, 2016) indicates the oral use of drugs. Table 3 represents the ADME properties predicted using SwissADME. Except for Hit_l, all the hits along with BQA show high GI absorption. Like Gl absorption, Blood brain barrier (BBB) is also considered an important factor for the distribution of 
drugs. Results show that only Hit_III and control are the BBB permeant among all hits. Thus, Hit_III can pass the BBB barrier and easily reach the neuron to target the PDZ domain of PICK1. Skin permeability of drugs is depicted by Log Kp. For the tested hits and control, Log Kp was observed as highly negative in the range of approximately $-6.5 \mathrm{~cm} / \mathrm{s}$ to $8.3 \mathrm{~cm} / \mathrm{s}$. The higher the negative value of Log Kp, the lesser the skin permeability, and this is used in Transdermal drug delivery (TDD) (Potts \& Guy, 1992). Furthermore, interactions of drugs with Cytochrome P450 (CYP) determines the drug elimination property through the metabolism of the drug (Testa \& Krämer, 2007). Our analysis suggests that all the hits and BQA showed inhibitory effects for the CYP3A4 isoform except Hit_III, whereas only Hit_II showed inhibition of CYP2C9 isoform. Permeability-glycoprotein (P-gp) is an important member of the ATP-dependent membrane transporter also known as ABC (ATP-binding cassette)-transporter which maintains the membrane transport effectively and protects CNS (Central nervous system) from the toxic components (Szakács et al., 2008). All the hits and control are predicted P-gp substrate.

\section{Bioavailability radar and BOILED-Egg model}

The bioavailability radar allows for a quick assessment of the compounds' drug-likeness. The pink area represents the optimal physicochemical region for each property that is expected to be orally bioavailable. LIPO (lipophilicity), SIZE (molecular weight), POLAR (polarity), INSOLU (insolubility), INSATU (insaturation), and FLEX (flexibility) are the six physicochemical parameters represented in the bioavailability radar plot, lipophilicity: XLOGP3 between -0.7 and +5.0 , size: molecular weight between the $150 \mathrm{~g} / \mathrm{mol}$ and $500 \mathrm{~g} / \mathrm{mol}$, polarity: TPSA (Topological polar surface area) between the $20 \AA^{2}$ and $130 \AA^{2}$, solubility: Log $S$ not higher than 6, saturation: fraction of carbons in the sp3 hybridization not less than 0.25 , and flexibility: no more than 9 rotatable bonds (Daina et al., 2017). From the bioavailability radar, it was observed that the Hit_ll obeys all the six rules. 


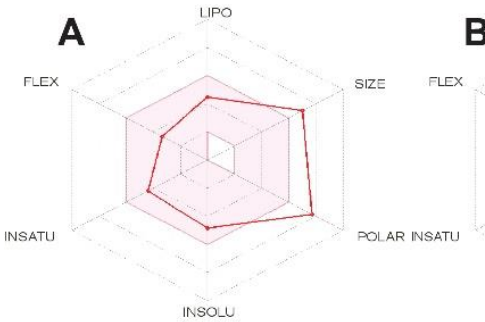

Hit_I
B

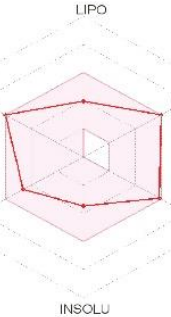

Hit_II

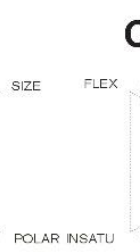

C

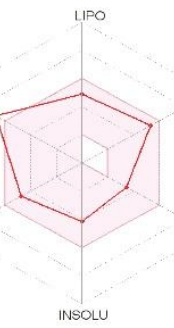

Hit_III

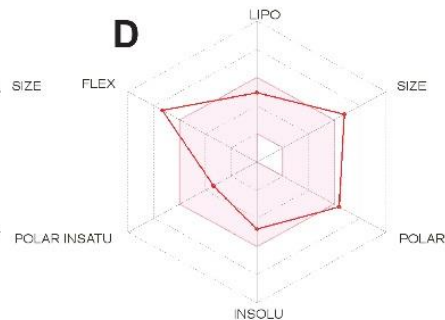

Hit_IV

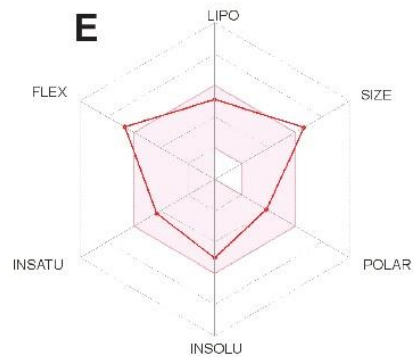

BQA

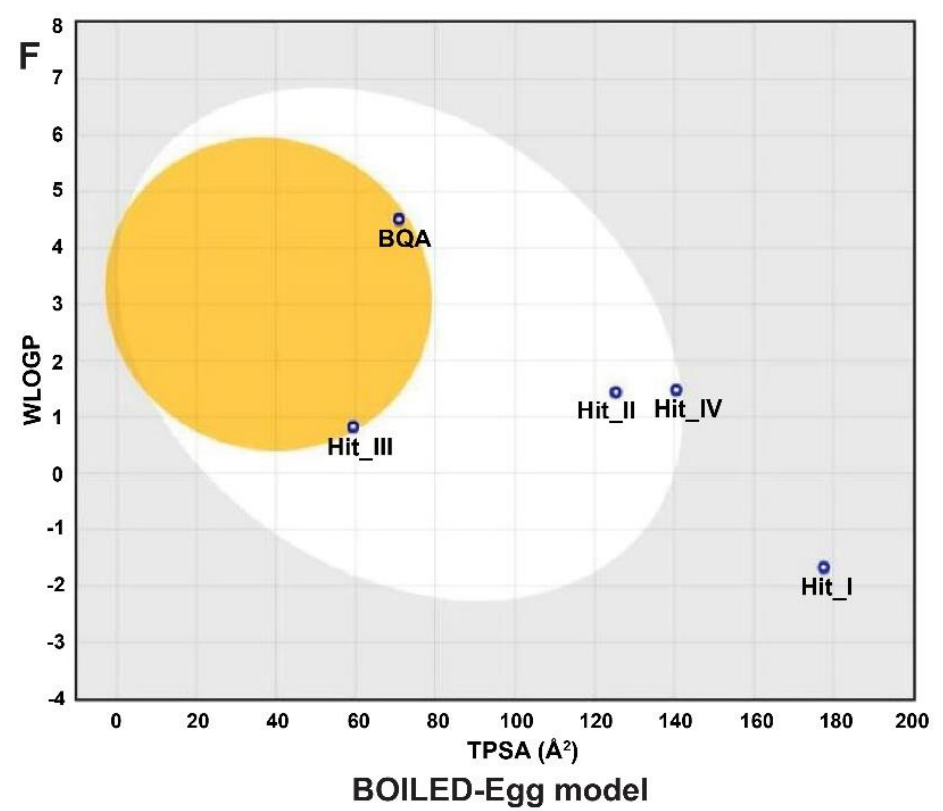

Figure 8. (A-E) The bioavailability radar charts of the Hit_I, Hit_II, Hit_III, Hit_IV, and control (BQA) respectively. (F) BOILEDEGG model for the four hits and BQA. Schematic representation of perceptive evaluation of passive Human intestinal absorption (HIA) and Blood brain barrier (BBB) with molecules in the WLOGP-versus-TPSA using BOILED-Egg model. The white part of the BOILED-Egg model indicates the high probability of HIA or GI tract passive absorption, whereas the yellow portion (yolk) indicates the high probability of BBB penetration. Furthermore, the dark blue circles are depicted for the substrate of P-glycoprotein (PGP+). Hit_I is not involved in the white or yellow regions which indicates the low GI absorption and BBB non-permeant whereas Hit_II and Hit_IV are observed to have high GI absorptions and BBB non-permeant. However, Hit_III and BQA (control) showed high GI absorption and BBB permeability.

Additionally, Hit_III also showed better agreement with the suggested rules except for FLEX while remaining violate the two to three rules. Furthermore, we included the BOILED-Egg model (Daina \& Zoete, 2016) to present a clear view of the drug-likeness of hits. Figure $8 \mathrm{~A}-\mathrm{E}$ and Figure $8 \mathrm{~F}$ illustrate the bioavailability radar and the BOILED-EGG model schematic diagram respectively for the studied compounds along with control.

\section{Conclusion}

To identify the potential hits for the PICK1 PDZ domain, pharmacophore-based virtual screening was performed using ten libraries containing 340,731,400 molecules and 1,603,779,177 conformers. Pharmacophore was constructed using a total of seven features. There were four potent hits identified from the different libraries. These hits were further subjected to molecular docking along with control inhibitor BQA. Docking results revealed that three hits, Hit_I (-9.0 $\mathrm{kcal} / \mathrm{mol})$, Hit_III $(-8.9 \mathrm{kcal} / \mathrm{mol})$, and Hit_IV $(-9.2 \mathrm{kcal} / \mathrm{mol})$ showed a slightly higher binding affinity with the PDZ domain in comparison with control $(-8.6 \mathrm{kcal} / \mathrm{mol})$ and Hit_IV was found to 
have the highest binding tendency $(-9.2 \mathrm{kcal} / \mathrm{mol})$ towards the PDZ domain. To probe the ligand binding and dynamic behaviour of the protein-ligand complexes, molecular dynamics simulation was performed. From the RMSD, RMSF, SASA, Rg, and hydrogen bond analyses, it was found that Hit_III allosterically impacts binding pocket on the PDZ domain but, showed large structural deviation in the allosteric pocket. However, Hit_Il was observed to show the lowest structural deviation in the PDZ binding pocket. Thus, Hit_II can be considered potential competitive inhibitor for the PDZ domain. Our results are a primary indication that Hit_Il efficiently binds with the PICK1 PDZ domain where the AMPA GluA2 peptide binds. Thus, Hit_II might inhibit and show potency against the PDZ domain but further experimental pieces of evidence are needed to verify this finding.

\section{Author contributions}

Shravan B. Rathod: Conceptualization, Investigation, Methodology, Data curation, Formal analysis, Writing-original draft, Writing-review \& editing. Pravin B. Prajapati: Investigation, Methodology, Data curation, Formal analysis, Writing-original draft, Writing-review \& editing. Ranjan Pal: Investigation, Methodology, Data curation, Formal analysis, Writing-original draft, Writing-review \& editing. Mohmedyasin F. Mansuri: Conceptualization, Investigation, Supervision, Methodology, Data curation, Formal analysis, Writing-original draft, Writing-review \& editing.

\section{Declaration of Competing Interest}

The authors declared no conflicts of interest.

\section{Acknowledgements}

SBR is thankful to his Chemistry Department for providing computational and infrastructure facilities.

\section{References}

Abraham, M. J., Murtola, T., Schulz, R., Páll, S., Smith, J. C., Hess, B., \& Lindah, E. (2015). Gromacs: High performance molecular simulations through multi-level parallelism from laptops to supercomputers. SoftwareX, 1-2, 19-25. https://doi.org/10.1016/j.softx.2015.06.001

Alfonso, S., Kessels, H. W., Banos, C. C., Chan, T. R., Lin, E. T., Kumaravel, G., Scannevin, R. H., Rhodes, K. J., Huganir, R., Guckian, K. M., Dunah, A. W., \& Malinow, R. (2014). Synaptodepressive effects of amyloid beta require PICK1. European Journal of Neuroscience, 39(7), 1225-1233. https://doi.org/10.1111/ejn.12499

Allouche, A. (2012). Software News and Updates Gabedit - A Graphical User Interface for Computational Chemistry Softwares. Journal of Computational Chemistry, 32, 174-182. https://doi.org/10.1002/jcc

Arkin, M. M. R., \& Wells, J. A. (2004). Small-molecule inhibitors of protein-protein interactions: Progressing towards the dream. In Nature Reviews Drug Discovery (Vol. 3, Issue 4, pp. $301-$ 317). https://doi.org/10.1038/nrd1343

Arnott, J. A., \& Planey, S. L. (2012). The influence of lipophilicity in drug discovery and design. Expert Opinion on Drug Discovery, 7(10), 863-875. https://doi.org/10.1517/17460441.2012.714363

Bach, A., Clausen, B. H., Møller, M., Vestergaard, B., Chi, C. N., Round, A., Sørensen, P. L., Nissen, K. B., Kastrup, J. S., Gajhede, M., Jemth, P., Kristensen, A. S., Lundström, P., Lambertsen, K. L., \& Strømgaard, K. (2012). A high-affinity, dimeric inhibitor of PSD-95 bivalently interacts with 
PDZ1-2 and protects against ischemic brain damage. Proceedings of the National Academy of Sciences of the United States of America, 109(9), 3317-3322.

https://doi.org/10.1073/pnas.1113761109

Bach, A., Stuhr-Hansen, N., Thorsen, T. S., Bork, N., Moreira, I. S., Frydenvang, K., Padrah, S., Christensen, S. B., Madsen, K. L., Weinstein, H., Gether, U., \& Strømgaard, K. (2010). Structure-activity relationships of a small-molecule inhibitor of the PDZ domain of PICK1. Organic and Biomolecular Chemistry, 8(19), 4281-4288. https://doi.org/10.1039/c0ob00025f

Bell, J. D., Park, E., Ai, J., \& Baker, A. J. (2009). PICK1-mediated GluR2 endocytosis contributes to cellular injury after neuronal trauma. Cell Death and Differentiation, 16(12), 1665-1680. https://doi.org/10.1038/cdd.2009.106

Bellone, C., \& Lüscher, C. (2006). Cocaine triggered AMPA receptor redistribution is reversed in vivo by mGluR-dependent long-term depression. Nature Neuroscience, 9(5), 636-641. https://doi.org/10.1038/nn1682

Berendsen, H. J. C., Postma, J. P. M., Van Gunsteren, W. F., Dinola, A., \& Haak, J. R. (1984). Molecular dynamics with coupling to an external bath. The Journal of Chemical Physics, 81(8), 3684-3690. https://doi.org/10.1063/1.448118

Berg, T. (2008). Small-molecule inhibitors of protein-protein interactions. Current Opinion in Drug Discovery \& Development, 11(5), 666-674. https://europepmc.org/article/med/18729018

Bergström, C. A. S., \& Larsson, P. (2018). Computational prediction of drug solubility in water-based systems: Qualitative and quantitative approaches used in the current drug discovery and development setting. International Journal of Pharmaceutics, 540(1-2), 185-193. https://doi.org/10.1016/j.jpharm.2018.01.044

Best, R. B., Zhu, X., Shim, J., Lopes, P. E. M., Mittal, J., Feig, M., \& MacKerell, A. D. (2012). Optimization of the additive CHARMM all-atom protein force field targeting improved sampling of the backbone $\varphi, \psi$ and side-chain $\times 1$ and $\times 2$ Dihedral Angles. Journal of Chemical Theory and Computation, 8(9), 3257-3273. https://doi.org/10.1021/ct300400x

Blazer, L. L., \& Neubig, R. R. (2009). Small molecule protein-protein interaction inhibitors as CNS therapeutic agents: Current progress and future hurdles. Neuropsychopharmacology, 34(1), 126-141. https://doi.org/10.1038/npp.2008.151

Chen, C., Huang, Y., Ji, X., \& Xiao, Y. (2013). Efficiently finding the minimum free energy path from steepest descent path. Journal of Chemical Physics, 138(16), 1-9. https://doi.org/10.1063/1.4799236

Cheng, T., Zhao, Y., Li, X., Lin, F., Xu, Y., Zhang, X., Li, Y., Wang, R., \& Lai, L. (2007). Computation of octanol-water partition coefficients by guiding an additive model with knowledge. Journal of Chemical Information and Modeling, 47(6), 2140-2148. https://doi.org/10.1021/ci700257y

Christensen, N. R., Čalyševa, J., Fernandes, E. F. A., Lüchow, S., Clemmensen, L. S., HaugaardKedström, L. M., \& Strømgaard, K. (2019). PDZ Domains as Drug Targets. Advanced Therapeutics, 2(7), 1-36. https://doi.org/10.1002/adtp.201800143

Constantinescu, T., Lungu, C. N., \& Lung, I. (2019). Lipophilicity as a central component of drug-like properties of chalchones and flavonoid derivatives. Molecules, 24(8), 1-11. https://doi.org/10.3390/molecules24081505

Daina, A., Michielin, O., \& Zoete, V. (2014). iLOGP: A Simple, Robust, and Efficient Description of n-Octanol/Water Partition Coefficient for Drug Design Using the GB/SA Approach. Journal of Chemical Information and Modeling, 54(12), 3284-3301. https://doi.org/10.1021/ci500467k

Daina, A., Michielin, O., \& Zoete, V. (2017). SwissADME: A free web tool to evaluate pharmacokinetics, drug-likeness and medicinal chemistry friendliness of small molecules. Scientific Reports, 7(March), 1-13. https://doi.org/10.1038/srep42717

Daina, A., \& Zoete, V. (2016). A BOILED-Egg To Predict Gastrointestinal Absorption and Brain Penetration of Small Molecules. ChemMedChem, 1117-1121. https://doi.org/10.1002/cmdc.201600182

Darden, T., York, D., \& Pedersen, L. (1993). Particle mesh Ewald: An N.log(N) method for Ewald sums in large systems. The Journal of Chemical Physics, 98(12), 10089-10092. https://doi.org/10.1063/1.464397 
Daw, M. I., Chittajallu, R., Bortolotto, Z. A., Dev, K. K., Duprat, F., Henley, J. M., Collingridge, G. L., \& Isaac, J. T. R. (2000). PDZ proteins interacting with C-terminal GluR2/3 are involved in a PKCdependent regulation of AMPA receptors at hippocampal synapses. Neuron, 28(3), 873-886. https://doi.org/10.1016/S0896-6273(00)00160-4

Delaney, J. S. (2004). ESOL: Estimating aqueous solubility directly from molecular structure. Journal of Chemical Information and Computer Sciences, 44(3), 1000-1005. https://doi.org/10.1021/ci034243x

Dev, K. K. (2004). Making protein interactions druggable: Targeting PDZ domains. Nature Reviews Drug Discovery, 3(12), 1047-1056. https://doi.org/10.1038/nrd1578

Dixon, R. M., Mellor, J. R., \& Hanley, J. G. (2009). PICK1-mediated glutamate receptor subunit 2 (GluR2) trafficking contributes to cell death in oxygen/glucose-deprivedhippocampal neurons. Journal of Biological Chemistry, 284(21), 14230-14235. https://doi.org/10.1074/jbc.M901203200

Ducki, S., \& Bennett, E. (2009). Protein-Protein Interactions: Recent Progress in the Development of Selective PDZ Inhibitors. Current Chemical Biology, 3(2), 146-158. https://doi.org/10.2174/2212796810903020146

Erlendsson, S., Rathje, M., Heidarsson, P. O., Poulsen, F. M., Madsen, K. L., Teilum, K., \& Gether, U. (2014). Protein interacting with C-kinase 1 (PICK1) binding promiscuity relies on unconventional PSD-95/discs-large/ZO-1 homology (PDZ) binding modes for nonclass II PDZ ligands. Journal of Biological Chemistry, 289(36), 25327-25340. https://doi.org/10.1074/jbc.M114.548743

Gardner, S. M., Takamiya, K., Xia, J., Suh, J. G., Johnson, R., Yu, S., \& Huganir, R. L. (2005). Calcium-permeable AMPA receptor plasticity is mediated by subunit-specific interactions with PICK1 and NSF. Neuron, 45(6), 903-915. https://doi.org/10.1016/j.neuron.2005.02.026

Garry, E. M., Moss, A., Rosie, R., Delaney, A., Mitchell, R., \& Fleetwood-Walker, S. M. (2003). Specific involvement in neuropathic pain of AMPA receptors and adapter proteins for the GluR2 subunit. Molecular and Cellular Neuroscience, 24(1), 10-22. https://doi.org/10.1016/S10447431(03)00134-9

Halgren, T. A. (1996). Performance of MMFF94*. Journal of Computational Chemistry, 17, 490-519. $\mathrm{http}: / / j o u r n a l s . w i l e y . c o m / j c c$

Hanley, J. G., \& Henley, J. M. (2005). PICK1 is a calcium-sensor for NMDA-induced AMPA receptor trafficking. EMBO Journal, 24(18), 3266-3278. https://doi.org/10.1038/sj.emboj.7600801

Hanwell, M. D., Curtis, D. E., Lonie, D. C., Vandermeersch, T., Zurek, E., \& Hutchison GR. (2012). Avogadro: an advanced semantic chemical editor, visualization, and analysis platform. Journal of Cheminformatics, 4(1), 1-17. https://doi.org/10.1186/1758-2946-4-17

Herlo, R., Lund, V. K., Lycas, M. D., Jansen, A. M., Khelashvili, G., Andersen, R. C., Bhatia, V., Pedersen, T. S., Albornoz, P. B. C., Johner, N., Ammendrup-Johnsen, I., Christensen, N. R., Erlendsson, S., Stoklund, M., Larsen, J. B., Weinstein, H., Kjærulff, O., Stamou, D., Gether, U., \& Madsen, K. L. (2018). An Amphipathic Helix Directs Cellular Membrane Curvature Sensing and Function of the BAR Domain Protein PICK1. Cell Reports, 23(7), 2056-2069. https://doi.org/10.1016/j.celrep.2018.04.074

Hess, B., Bekker, H., Berendsen, H. J. C., \& Fraaije, J. G. E. M. (1997). LINCS: A Linear Constraint Solver for molecular simulations. Journal of Computational Chemistry, 18(12), 1463-1472. https://doi.org/10.1002/(SICl)1096-987X(199709)18:12<1463::AID-JCC4>3.0.CO;2-H

Houslay, M. D. (2009). Disrupting specific PDZ domain-mediated interactions for therapeutic benefit. British Journal of Pharmacology, 158(2), 483-485. https://doi.org/10.1111/j.14765381.2009.00359.x

Hsieh, H., Boehm, J., Sato, C., Iwatsubo, T., Tomita, T., Sisodia, S., \& Malinow, R. (2006). AMPAR Removal Underlies A $\beta$-Induced Synaptic Depression and Dendritic Spine Loss. Neuron, 52(5), 831-843. https://doi.org/10.1016/j.neuron.2006.10.035

Jang, G. R., Harris, R. Z., \& Lau, D. T. (2001). Pharmacokinetics and its role in small molecule drug discovery research. Medicinal Research Reviews, 21(5), 382-396. https://doi.org/10.1002/med.1015

Johnson, A. W. (1998). Invitation to Organic Chemistry. Jones \& Bartlett Publishers, Inc (Verlag). pp243. 
Jorgensen, W. L., Chandrasekhar, J., Madura, J. D., Impey, R. W., \& Klein, M. L. (1983). Comparison of simple potential functions for simulating liquid water. The Journal of Chemical Physics, 79(2), 926-935. https://doi.org/10.1063/1.445869

Khan, Z., \& Lafon, M. (2014). PDZ domain-mediated protein interactions: therapeutic targets in neurological disorders. Current Medicinal Chemistry, 21(23), 2632-2641. https://doi.org/10.2174/0929867321666140303145312

Kim, C. H., Hee Jung Chung, Lee, H. K., \& Huganir, R. L. (2001). Interaction of the AMPA receptor subunit GluR2/3 with PDZ domains regulates hippocampal long-term depression. Proceedings of the National Academy of Sciences of the United States of America, 98(20), 11725-11730. https://doi.org/10.1073/pnas.211132798

Kim, E., Niethammer, M., Rothschild, A. Jan, Y. N., \& Sheng, M. (1995). Clustering of Shaker-type K+ channels by interaction with a family of membrane-associated guanylate kinases. Nature, 378, 85-88. https://doi.org/10.1038/378085a0

Kornau, H., Schenker, L. T., Kennedy, M. B., \& Seeburg, P. H. (1993). 4-S]s variant expression appears to be develop-mentally regulated, with the $\mathrm{Cl}$ cassette-containing splice variants exhibiting in. 28-31. www.sciencemag.org

Li, Y. H., Zhang, N., Wang, Y. N., Shen, Y., \& Wang, Y. (2016). Multiple faces of protein interacting with C kinase 1 (PICK1): Structure, function, and diseases. Neurochemistry International, 98, 115-121. https://doi.org/10.1016/j.neuint.2016.03.001

Lin, E. Y. S., Silvian, L. F., Marcotte, D. J., Banos, C. C., Jow, F., Chan, T. R., Arduini, R. M., Qian, F., Baker, D. P., Bergeron, C., Hession, C. A., Huganir, R. L., Borenstein, C. F., Enyedy, I., Zou, J., Rohde, E., Wittmann, M., Kumaravel, G., Rhodes, K. J., ... Guckian, K. M. (2018). Potent PDZDomain PICK1 Inhibitors that Modulate Amyloid Beta-Mediated Synaptic Dysfunction. Scientific Reports, 8(1), 1-10. https://doi.org/10.1038/s41598-018-31680-3

Lipinski, C. A., Lombardo, F., Dominy, B. W., \& Feeney, P. J. (2012). Experimental and computational approaches to estimate solubility and permeability in drug discovery and development settings. Advanced Drug Delivery Reviews, 64(SUPPL.), 4-17. https://doi.org/10.1016/j.addr.2012.09.019

Lippert, R. A., Bowers, K. J., Dror, R. O., Eastwood, M. P., Gregersen, B. A., Klepeis, J. L., Kolossvary, I., \& Shaw, D. E. (2007). A common, avoidable source of error in molecular dynamics integrators. Journal of Chemical Physics, 126(4), 2006-2008. https://doi.org/10.1063/1.2431176

MacKerell, A. D., Bashford, D., Bellott, M., Dunbrack, R. L., Evanseck, J. D., Field, M. J., Fischer, S., Gao, J., Guo, H., Ha, S., Joseph-McCarthy, D., Kuchnir, L., Kuczera, K., Lau, F. T. K., Mattos, C., Michnick, S., Ngo, T., Nguyen, D. T., Prodhom, B., ... Karplus, M. (1998). All-atom empirical potential for molecular modeling and dynamics studies of proteins. Journal of Physical Chemistry B, 102(18), 3586-3616. https://doi.org/10.1021/jp973084f

Marcotte, D. J., Hus, J. C., Banos, C. C., Wildes, C., Arduini, R., Bergeron, C., Hession, C. A., Baker, D. P., Lin, E., Guckian, K. M., Dunah, A. W., \& Silvian, L. F. (2018). Lock and chop: A novel method for the generation of a PICK1 PDZ domain and piperidine-based inhibitor co-crystal structure. Protein Science, 27(3), 672-680. https://doi.org/10.1002/pro.3361

Marsault, E., Benakli, K., Beaubien, S., Saint-Louis, C., Déziel, R., \& Fraser, G. (2007). Potent macrocyclic antagonists to the motilin receptor presenting novel unnatural amino acids. Bioorganic and Medicinal Chemistry Letters, 17(15), 4187-4190. https://doi.org/10.1016/j.bmcl.2007.05.043

Martin, Y. C. (2005). A bioavailability score. Journal of Medicinal Chemistry, 48(9), 3164-3170. https://doi.org/10.1021/jm0492002

Moriguchi, I., Hirono, S., Liu, Q., Nakagome, I., \& Matsushita, Y. (1992). Simple Method of Calculating Octanol/Water Partition Coefficient. Chemical and Pharmaceutical Bulletin, 40(1), 127-130. https://doi.org/10.1248/cpb.40.127

Pan, L., Wu, H., Shen, C., Shi, Y., Jin, W., Xia, J., \& Zhang, M. (2007). Clustering and synaptic targeting of PICK1 requires direct interaction between the PDZ domain and lipid membranes. EMBO Journal, 26(21), 4576-4587. https://doi.org/10.1038/sj.emboj.7601860

Pant, R., Joshi, A., Maiti, P., Nand, M., Pande, V., \& Chandra, S. (2020). Identification of potential Mycolyltransferase Ag85C inhibitors of Mycobacterium tuberculosis H37Rv via Virtual High 
Throughput Screening and Binding free energy studies. Journal of Molecular Graphics and Modelling, 98, 107584. https://doi.org/10.1016/j.jmgm.2020.107584

Parrinello, M., \& Rahman, A. (1981). Polymorphic transitions in single crystals: A new molecular dynamics method. Journal of Applied Physics, 52(12), 7182-7190.

https://doi.org/10.1063/1.328693

Pires, D. E. V., Blundell, T. L., \& Ascher, D. B. (2015). pkCSM: Predicting small-molecule pharmacokinetic and toxicity properties using graph-based signatures. Journal of Medicinal Chemistry, 58(9), 4066-4072. https://doi.org/10.1021/acs.jmedchem.5b00104

Potts, R.O., \& Guy, R.H. (1992). Predicting Skin Permeability. Pharmaceutical Research, 9(5), 663669. https://doi.org/10.1023/A:1015810312465

Santos, K. B., Guedes, I. A., Karl, A. L. M., \& Dardenne, L. E. (2020). Highly Flexible Ligand Docking: Benchmarking of the DockThor Program on the LEADS-PEP Protein-Peptide Data Set. Journal of Chemical Information and Modeling, 60(2), 667-683. https://doi.org/10.1021/acs.jcim.9b00905

Savjani, K. T., Gajjar, A. K., \& Savjani, J. K. (2012). Drug Solubility: Importance and Enhancement Techniques. ISRN Pharmaceutics, 2012(100 mL), 1-10. https://doi.org/10.5402/2012/195727

Schrödinger LLC. (2010). The PyMOL molecular graphics system. Version 2.4.1

Schyman, P., Liu, R., Desai, V., \& Wallqvist, A. (2017). vNN web server for ADMET predictions. Frontiers in Pharmacology, 8(DEC), 1-14. https://doi.org/10.3389/fphar.2017.00889

Sharma, T., \& Siddiqi, M. I. (2019). In silico identification and design of potent peptide inhibitors against PDZ-3 domain of Postsynaptic Density Protein (PSD-95). Journal of Biomolecular Structure and Dynamics, 37(5), 1241-1253. https://doi.org/10.1080/07391102.2018.1454851

Singh, R., Ganeshpurkar, A., Kumar, D., Kumar, D., Kumar, A., \& Singh, S. K. (2020). Identifying potential GluN2B subunit containing N-Methyl-D-aspartate receptor inhibitors: an integrative in silico and molecular modeling approach. Journal of Biomolecular Structure and Dynamics, 38(9), 2533-2545. https://doi.org/10.1080/07391102.2019.1635530

Songyang, Z., Fanning, A. S., Fu, C., Xu, J., Marfatia, S. M., Chishti, A. H., Crompton, A., Chan, A. C., Anderson, J. M., \& Cantley, L. C. (1997). Recognition of unique carboxyl-terminal motifs by distinct PDZ domains. Science, 275(5296):73-77. doi: 10.1126/science.275.5296.73

Sunseri, J., \& Koes, D. R. (2016). Pharmit: interactive exploration of chemical space. Nucleic Acids Research, 44(W1), W442-W448. https://doi.org/10.1093/nar/gkw287

Szakács, G., Váradi, A., Özvegy-Laczka, C., \& Sarkadi, B. (2008). The role of ABC transporters in drug absorption, distribution, metabolism, excretion and toxicity (ADME-Tox). Drug Discovery Today, 13(9-10), 379-393. https://doi.org/10.1016/j.drudis.2007.12.010

Terashima, A., Pelkey, K. A., Rah, J. C., Suh, Y. H., Roche, K. W., Collingridge, G. L. L., McBain, C. J., \& Isaac, J. T. R. (2008). An Essential Role for PICK1 in NMDA Receptor-Dependent Bidirectional Synaptic Plasticity. Neuron, 57(6), 872-882.

https://doi.org/10.1016/j.neuron.2008.01.028

Testa, B., \& Krämer, S. D. (2007). The biochemistry of drug metabolism - An introduction part 2. Redox reactions and their enzymes. Chemistry and Biodiversity, 4(3), 257-405. https://doi.org/10.1002/cbdv.200790032

Thorsen, T. S., Madsen, K. L., Rebola, N., Rathje, M., Anggono, V., Bach, A., Moreira, I. S., StuhrHansen, N., Dyhring, T., Peters, D., Beuming, T., Huganir, R., Weinstein, H., Mulle, C., Strømgaard, K., Rønn, L. C. B., \& Gether, U. (2010). Identification of a small-molecule inhibitor of the PICK1 PDZ domain that inhibits hippocampal LTP and LTD. Proceedings of the National Academy of Sciences of the United States of America, 107(1), 413-418. https://doi.org/10.1073/pnas.0902225107

Thorsen, T., S. Madsen, K., Dyhring, T., Bach, A., Peters, D., Stromgaard, K., \& Gether, U. (2011). A Fluorescence Polarization Based Screening Assay for Identification of Small Molecule Inhibitors of the PICK1 PDZ Domain. Combinatorial Chemistry \& High Throughput Screening, 14(7), 590600. https://doi.org/10.2174/138620711796367201

Velázquez-Libera, J. L., Durán-Verdugo, F., Valdés-Jiménez, A., Valdés-Jiménez, A., Núñez-Vivanco, G., \& Caballero, J. (2020). LigRMSD: A web server for automatic structure matching and RMSD 
calculations among identical and similar compounds in protein-ligand docking. Bioinformatics, 36(9), 2912-2914. https://doi.org/10.1093/bioinformatics/btaa018

Verlet, L. (1967). Computer "Experiments" on Classical Fluids. I. Thermodynamical Properties of Lennard-Jones Molecules. Physical Review, 159(1), 98-103.

https://doi.org/10.1103/PhysRev.159.98

Wang, G., Zhang, X., Pan, X., \& Xiao, Y. (2021). FSC231 can alleviate paclitaxel-induced neuralgia by inhibiting PICK1 and affecting related factors. Neuroscience Letters, 741(39), 135471. https://doi.org/10.1016/j.neulet.2020.135471

Webb, B., \& Sali, A. (2016). Comparative protein structure modeling using MODELLER. Current Protocols in Bioinformatics, 2016, 5.6.1-5.6.37. https://doi.org/10.1002/cpbi.3

Wells, J. A., \& McClendon, C. L. (2007). Reaching for high-hanging fruit in drug discovery at proteinprotein interfaces. Nature, 450(7172), 1001-1009. https://doi.org/10.1038/nature06526

Wildman, S. A., \& Crippen, G. M. (1999). Prediction of physicochemical parameters by atomic contributions. Journal of Chemical Information and Computer Sciences, 39(5), 868-873. https://doi.org/10.1021/ci990307|

Xia, J., Chung, H. J., Wihler, C., Huganir, R. L., \& Linden, D. J. (2000). Cerebellar long-term depression requires PKC-regulated interactions between GluR2/3 and PDZ domain-containing proteins. Neuron, 28(2), 499-510. https://doi.org/10.1016/S0896-6273(00)00128-8

Xu, J., \& Xia, J. (2007). Structure and function of PICK1. NeuroSignals, 15(4), 190-201. https://doi.org/10.1159/000098482

Yang, H., Sun, L., Wang, Z., Li, W., Liu, G., \& Tang, Y. (2018). ADMETopt: A Web Server for ADMET Optimization in Drug Design via Scaffold Hopping. Journal of Chemical Information and Modeling, 58(10), 2051-2056. https://doi.org/10.1021/acs.jcim.8b00532

Yu, W., He, X., Vanommeslaeghe, K., \& MacKerell, A. D. (2012). Extension of the CHARMM general force field to sulfonyl-containing compounds and its utility in biomolecular simulations. Journal of Computational Chemistry, 33(31), 2451-2468. https://doi.org/10.1002/jcc.23067

Zhou, P., Jin, B., Li, H., \& Huang, S. Y. (2018). HPEPDOCK: A web server for blind peptide-protein docking based on a hierarchical algorithm. Nucleic Acids Research, 46(W1), W443-W450. https://doi.org/10.1093/nar/gky357 